\title{
Atherosclerosis and flow: roles of epigenetic modulation in vascular endothelium
}

\author{
Ding-Yu Lee ${ }^{1}$ and Jeng-Jiann Chiu ${ }^{2,3,4,5,6^{*}}$
}

\begin{abstract}
Background: Endothelial cell (EC) dysfunctions, including turnover enrichment, gap junction disruption, inflammation, and oxidation, play vital roles in the initiation of vascular disorders and atherosclerosis. Hemodynamic forces, i.e., atherprotective pulsatile (PS) and pro-atherogenic oscillatory shear stress (OS), can activate mechanotransduction to modulate EC function and dysfunction. This review summarizes current studies aiming to elucidate the roles of epigenetic factors, i.e., histone deacetylases (HDACs), non-coding RNAs, and DNA methyltransferases (DNMTs), in mechanotransduction to modulate hemodynamics-regulated EC function and dysfunction.
\end{abstract}

Main body of the abstract: OS enhances the expression and nuclear accumulation of class I and class II HDACs to induce EC dysfunction, i.e., proliferation, oxidation, and inflammation, whereas PS induces phosphorylationdependent nuclear export of class II HDACs to inhibit EC dysfunction. PS induces overexpression of the class III HDAC Sirt1 to enhance nitric oxide (NO) production and prevent EC dysfunction. In addition, hemodynamic forces modulate the expression and acetylation of transcription factors, i.e., retinoic acid receptor a and krüppel-like factor-2, to transcriptionally regulate the expression of microRNAs (miRs). OS-modulated miRs, which stimulate proliferative, pro-inflammatory, and oxidative signaling, promote EC dysfunction, whereas PS-regulated miRs, which induce antiproliferative, anti-inflammatory, and anti-oxidative signaling, inhibit EC dysfunction. PS also modulates the expression of long non-coding RNAs to influence EC function. i.e., turnover, aligmant, and migration. On the other hand, OS enhances the expression of DNMT-1 and -3a to induce EC dysfunction, i.e., proliferation, inflammation, and NO repression.

Conclusion: Overall, epigenetic factors play vital roles in modulating hemodynamic-directed EC dysfunction and vascular disorders, i.e., atherosclerosis. Understanding the detailed mechanisms through which epigenetic factors regulate hemodynamics-directed EC dysfunction and vascular disorders can help us to elucidate the pathogenic mechanisms of atherosclerosis and develop potential therapeutic strategies for atherosclerosis treatment.

Keywords: DNA methyltransferase, Endothelial cell, Epigenetic factor, Hemodynamic force, Histone deacetylase, Non-coding RNA

\section{Introduction}

Vascular endothelial cells (ECs), which are located in the blood vessel wall and function to prevent vascular leakage and protect vascular vessels, are subjected to hemodynamic forces that can activate mechanotransduction and regulate

\footnotetext{
* Correspondence: jjchiu@nhri.org.tw

${ }^{2}$ Institute of Cellular and System Medicine, National Health Research Institutes, Miaoli 350, Taiwan

${ }^{3}$ Institute of Biomedical Engineering, National Tsing Hua University, Hsinchu 300, Taiwan

Full list of author information is available at the end of the article
}

homeostasis. Pro-atherogenic oscillatory shear stress (OS) and atheroprotective pulsatile shear stress (PS) are two vital hemodynamic forces that modulate EC dysfunction and function [1-3]. Pro-atherogenic OS serves as "bad flow" to activate various pro-atherogenic signaling pathways and gene expression, resulting in promotion of pathogenic conditions in ECs. In contrast, atheroprotective PS serves as "good flow" to induce many protective signaling pathways and gene expression, thereby maintaining normal physiological functions in ECs [1-3]. In the aortic circulation system, pro-atherogenic OS preferentially occurs in the 
specific regions of aortic trees, i.e., the inner curvatures of the aortic arch; carotid bifurcations; branch points of the coronary, infrarenal, and femoral arteries; and aorto-renal branches. These OS regions have been identified as atherosclerosis-susceptible regions in the aortic system (Fig. 1) [1-3]. Atheroprotective PS usually develops in the straight segments of aortic tree, i.e., the descending thoracic aorta and distal straight renal artery. These PS regions have been identified as athero-protective regions in the aortic system (Fig. 1) [1-3].

EC dysfunctions, including turnover enhancement, gap junction disruption, inflammation, and oxidation, have been found to play vital roles in the initiation of vascular disorders and atherosclerosis [4-7]. Turnover enhancement and gap junction disruption in ECs are pathogenic factors for atherosclerosis $[1,7,8]$. These responses can disrupt the intact structure of the endothelium to increase endothelial permeability and allow the penetration of lipoproteins and inflammatory monocytes to promote the progression of atherosclerosis [5-8]. Proatherogenic OS and atheroprotective PS exert differential effects on modulating cell proliferation and gap junctions in ECs. Pro-atherogenic OS induces EC proliferation and increases turnover rates by inducing DNA synthesis and cyclin and cyclin-dependent kinase expression and inhibiting $\mathrm{p} 21_{\mathrm{CIP} 1}$ expression $[9,10]$. In contrast, PS inhibits EC proliferation via induction of $\mathrm{p} 21_{\mathrm{CIP1}}$ to inhibit DNA synthesis, resulting in EC arrest at the $G_{0} / G_{1}$ phase [11]. In vivo, ECs in disturbed flow regions with pro-atherogenic OS have higher mitotic rates than the ECs in the straight segments of aorta with atheroprotective PS in the rabbit thoracic aorta [12]. Guo et al. [13] demonstrated that the differential effects of atheroprotective PS and pro-atherogenic OS on the cell cycle are mediated by AKT/mammalian target of rapamycin $(\mathrm{mTOR}) / \mathrm{p} 70$ signaling pathways. Moreover, hemodynamic forces have been found to regulate junctional proteins, including connexins $(\mathrm{Cx})$ and vascular endothelial (VE)-cadherin, to regulate endothelial permeability $[14,15]$. Pro-atherogenic OS induces discontinuous distributions of VE-cadherin and $\beta$-catenin, whereas atheroprotective PS induces continuous distributions of these proteins [14]. Pro-atherogenic OS also induces discontinuous $\mathrm{Cx} 43$ at the EC periphery [15]. In vivo studies have further demonstrated that VE-cadherin is highly expressed at EC borders in the descending

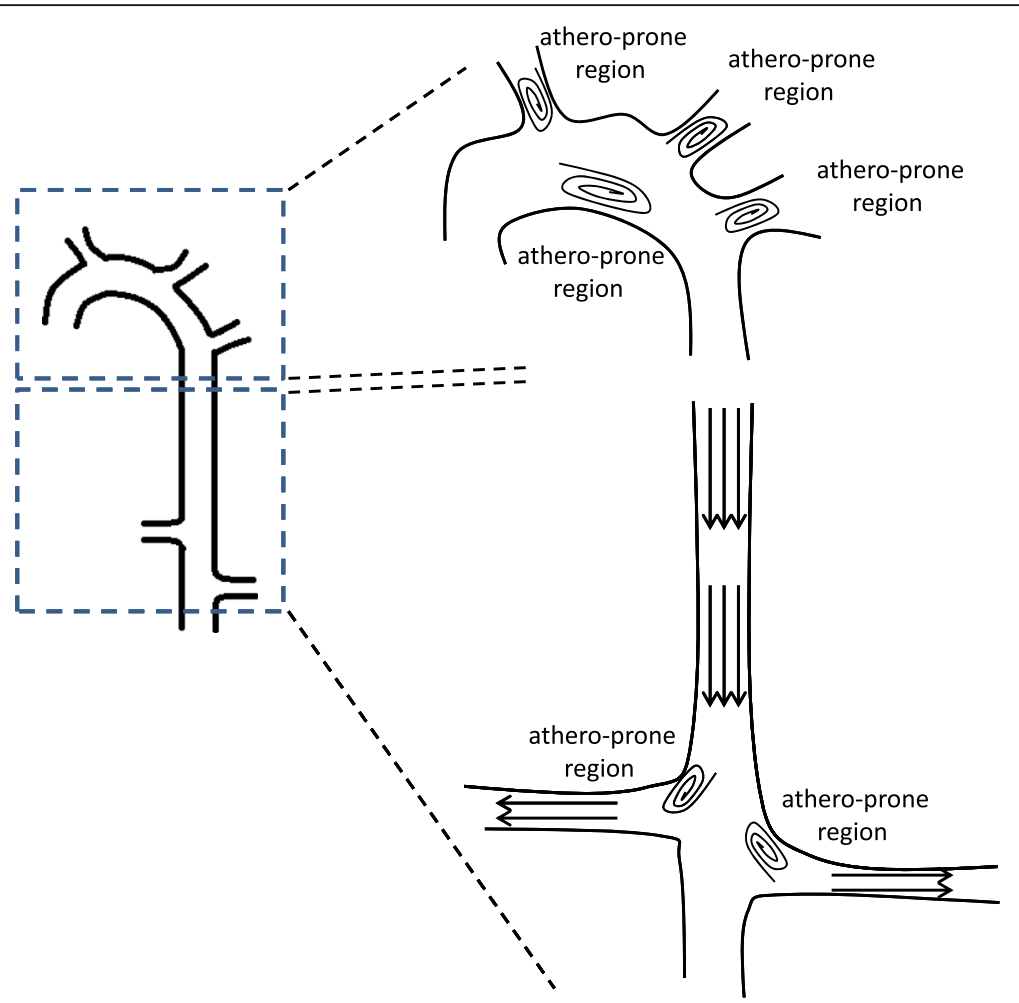

Fig. 1 Distribution of hemodynamic forces, i.e., pro-atherogenic OS and atheroprotective PS, in the aortic trees. Pro-atherogenic OS and atheroprotective PS are two types of hemodynamic forces to affect EC function or dysfunction. Pro-atherogenic OS develops in the atherosclerosis-susceptible regions of aortic tree, e.g., the inner curvatures of aortic arch; carotid bifurcations; branch points of the coronary, infrarenal, and femoral arteries; and aorto-renal branches. In contrast, atheroprotective PS occurs in the atherosclerosis-protective regions of aortic tree, e.g., descending thoracic aorta and distal straight renal artery. The athero-prone regions are labeled in the figure. $\vec{\longrightarrow}$ : pro-atherogenic OS; 
thoracic aorta (PS region), but is rarely expressed in the aortic arch (OS region) [14].

EC inflammation is an additional pathogenic factor for atherosclerosis $[4-7,16]$. ECs have been found to increase the expression of pro-inflammatory chemotactic molecules, e.g., monocyte chemoattractant protein-1 (MCP-1), and adhesion molecules, e.g., intercellular adhesion molecule-1 (ICAM-1), vascular cell adhesion molecule-1 (VCAM-1), and E-selectin/P-selectin, to recruit monocytes for adhesion to and penetration into vessel walls, thereby initiating the progression of atherosclerosis $[4-7,16]$. Pro-atherogenic OS and atheroprotective PS have opposite effects on modulating these pro-inflammatory genes to regulate EC dysfunction and function. Pro-atherogenic OS induces the sustained expression or activation of transcription factors, e.g., nuclear factor (NF)- $\mathrm{kB}$, to induce the expression of proinflammatory genes and activate atherogenic signaling in ECs. In contrast, atheroprotective PS only transiently induces or even inhibits the expression of these pro-inflammatory genes and the activation of atherogenic signaling in ECs [1, 17-20].

EC oxidation is also a pathogenic factor for atherosclerosis [4-7]. ECs can modulate intracellular superoxide and antioxidant enzymes to regulate atherogenic responses in the progression of atherosclerosis [21]. Proatherogenic OS induces a sustained increase of intracellular superoxide to enhance oxidative stress or reactive oxygen species (ROS) levels to damage blood vessels, whereas atheroprotective PS induces antioxidant enzymes, i.e., superoxide dismutase (SOD), heme oxygenase- 1 , and $\mathrm{NADPH}$ quinine oxidoreductase 1 (NQO1), to protect blood vessels $[22,23]$. In addition to the modulatory effects of atheroprotective PS and pro-atherogenic OS on EC proliferation, inflammation, and oxidation, atheroprotective PS also activates protective signaling to maintain EC physiological function. PS induces the expression or activation of various atheroprotective signaling molecules, including endothelial nitric oxide synthase (eNOS), prostaglandin $\mathrm{I}_{2}$, nitric oxide $(\mathrm{NO})$, glutathione peroxidase (Gpx), and glutathione reductase [1, 24, 25]. Taken together, these studies suggest that hemodynamic forces, i.e., pro-atherogenic OS and atheroprotective PS, exert differential effects on modulating EC function and dysfunction, subsequently regulating disease and health.

Epigenetic modulation is defined as any stable and heritable change in gene expression or cellular function without changes in DNA sequences [26]. These modifications, which include covalent and noncovalent modifications of DNA, as well as histone tails, affect changes in chromatin structure and gene expression. DNA deacetylation, DNA methylation, and RNA-based mechanisms are the three main forms of epigenetic modulation. DNA deacetylation and methylation are catalyzed by specific enzymes, i.e., histone deacetylases (HDACs) and DNA methyltransferases (DNMTs), to regulate gene expression. RNA-based mechanisms are directed by noncoding RNAs to regulate gene expression [27-31]. Recent studies have shown that these epigenetic factors, including HDACs [27], non-coding RNAs [28-30], and DNMTs [31], play vital roles in epigenetic regulations of vascular function and dysfunction (Fig. 2).

This review focus on discussing (1) the effects of hemodynamic forces, i.e., OS and PS, on modulating the expression and activation of epigenetic factors in ECs and (2) the roles of hemodynamics-modulated epigenetic factors in regulating mechanotransduction, including signaling molecules, transcription factors, and gene expression, involved in atherogenic and atheroprotective signaling in ECs. Moreover, the relationship between hemodynamics-modulated epigenetic factors and vascular disorders is also discussed. This article summarizes the evidence that epigenetic factors are vital factors for modulating hemodynamics-directed EC function and dysfunction, and provides insights into the functional roles of epigenetic factors in the development of atherosclerosis in responses to flow.

\section{HDACs in hemodynamics-directed EC function and dysfunction \\ Classification and function of HDACs}

Histone deacetylation is a common modification affecting chromosome packaging and DNA transcription. HDACs are specific enzymes that function to deacetylate the $\varepsilon$ - $\mathrm{N}$-acetyl lysine amino acid on histones to modulate the chromatin structure involved in DNA replication or silencing [32]. Hyperacetylation is related to upregulation of transcription, whereas hypoacetylation is associated with downregulation of transcription [32]. In addition to histone deacetylation, HDACs also interact with nonhistone proteins, i.e., various signaling molecules and transcription factors, to repress their functions [27]. Because most functions of HDACs, i.e., histone deacetylation and transcription factor repression, are processed only in the nucleus, the localization of HDACs is vital for evaluating their functions [27]. HDACs in mammalian cells are classified into four groups depending on their sequence similarity: class I HDACs (HDAC-1, $-2,-3$, and -8 ), class II HDACs (IIa: HDAC-4, $-5,-7$, and -9 , and IIb: HDAC-6, -10 ), class III HDACs (sirtuins (Sirts): Sirt1-7), and class IV HDACs (HDAC-11). Classes I, II, and IV HDACs are zinc-dependent enzymes, whereas class III HDACs are NAD + -dependent enzymes [33-35].

Class I HDACs are nuclear enzymes. HDAC-1, -2, and -8 are generally found only in the nucleus, whereas HDAC-3 shuttles between the nucleus and cytoplasm [33-35]. Inhibition studies have demonstrated that class 

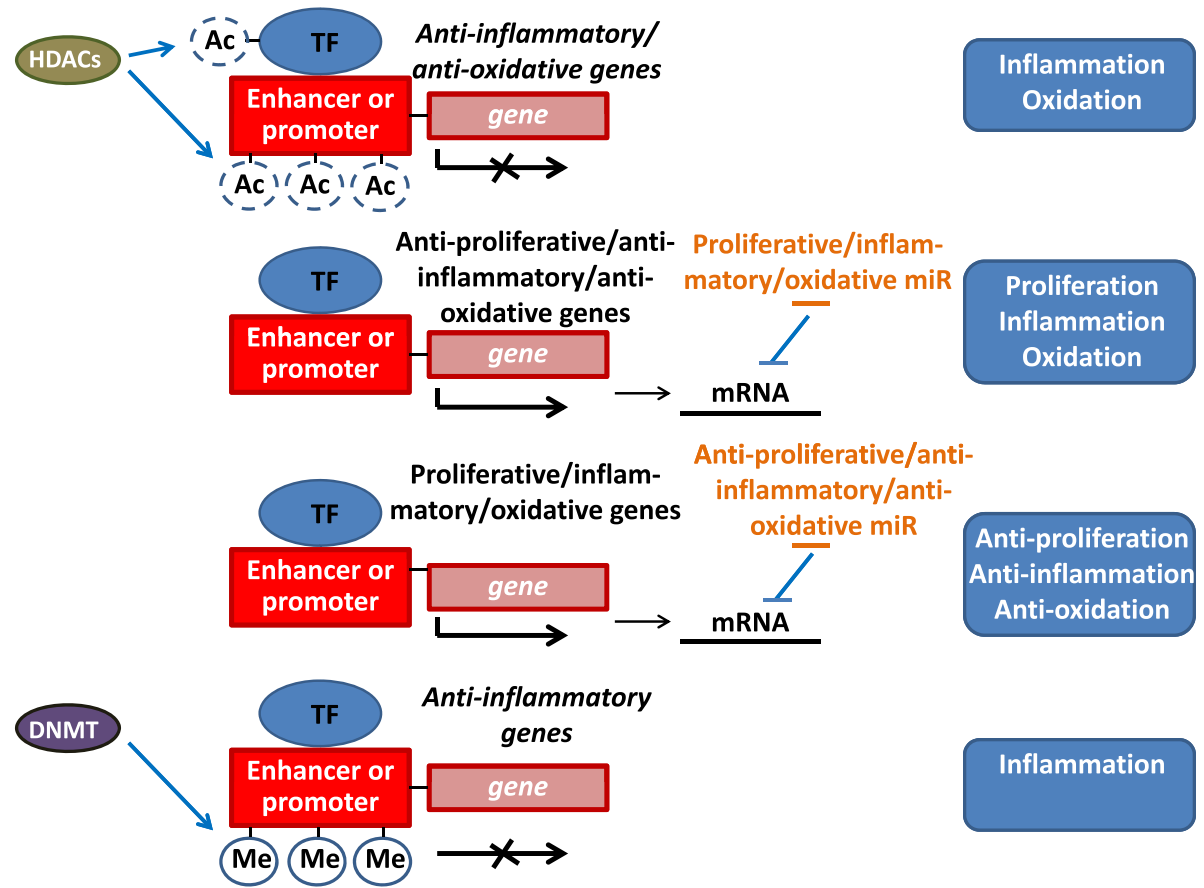

Inflammation

Fig. 2 Epigenetic regulation of HDACs, non-coding RNAs, and DNMTs in vascular function and dysfunction. HDACs deacetylate not only the transcriptional factor, but also DNA regions of promoter or enhancer, to repress the expressions of anti-inflammatory or anti-oxidative genes. Proliferative, oxidative, and pro-inflammatory miRs target anti-proliferative, anti-oxidative, and anti-inflammatory mRNAs to drive proliferative, oxidative, and inflammatory signaling, respectively. In contrast, anti-proliferative, anti-oxidative, and anti-inflammatory miRs target the respective mRNAs to drive anti-proliferative, anti-oxidative, and anti-inflammatory signaling, respectively. DNMTs methylate DNA regions of promoter or enhancer to inhibit the expression of anti-inflammatory genes to elicit inflammatory signaling. Ac: acetylation; TF: transcription factor; Me: methylation

I HDACs play vital roles in modulating cell proliferation and survival [33-35]. HDAC-1 and -2 can be recruited by transcription factors, including Sp1, Sp3, p53, NF- $\mathrm{kB}$, and YY1, to form the multiprotein corepressor Sin3, nucleosome-remodeling HDAC, and CoREST [33-35], which repress the transcriptional activity and cellular functions of these transcription factors. HDAC-3 is involved in two complexes, i.e., nuclear receptor corepressor (NCoR or NCOR1) and silencing mediator of retinoic acid and thyroid hormone receptor (SMRT or NCOR2) complexes. NCoR and SMRT further recruit class II HDACs as bridges for HDAC-3 to enhance NCoR/SMRT/HDAC-3 activity and repress specific transcription factors, i.e., myocyte enhancer factor 2 (MEF2), which modulates MEF2-directed cell functions [33-35]. In addition, the phosphorylation of HDAC-1 (at S393, S421, and S423), HDAC-2 (at S394, S422, and S424), and HDAC-3 (at S424) can enhance their activity and further repress transcription factor function [33-35].

Class II HDACs are located in both the nucleus and cytosol and can be phosphorylated to regulate their nuclear/cytosolic shuttling [36, 37]. The functions of class II HDACs are related to cell inflammation and migration. As described above, class IIa HDACs (HDAC-4, $5,-7$, and -9) can collaborate with NCoR/SMRT/
HDAC-3 to repress MEF2 transcriptional activity and related cellular functions, including inflammation. In addition to MEF2, class IIa HDACs can also associate with other transcription factors to repress their transcriptional activity [33-35]. Moreover, class IIa HDACs can be phosphorylated to enhance their binding to 143-3 proteins, induce nuclear export, and rescue the repression of transcription factors and downstream genes. Thus, nuclear-cytoplasmic shuttling of class IIa HDACs can be regulated by various kinases and phosphatases involved in signaling transduction to modulate the functions of class IIa HDACs and various transcription factors [36, 37]. Class IIb HDACs (HDAC-6 and -10) also shuttle between the nucleus and cytoplasm, but exist primarily in the cytoplasm [33-35]. HDAC-6 functions as a $\alpha$-tubulin or cortactin deacetylase to regulate microtubule- and actin-dependent cell motility. Moreover, HDAC-6 can form aggresomes to clear misfolded proteins [33-35, 38].

Class III HDACs (Sirt1-7) can transfer an acetyl group from lysine to the cofactor nicotinamide adenine dinucleotide $(\mathrm{NAD}+)$ to generate $\mathrm{O}$-acetyl ADP-ribose and nicotinamide, which serve as feedback inhibitors of the enzymatic reaction. Sirt1, 6, and 7 locate in the nucleus, Sirt3, 4, and 5 locate in the micochondria, and 
Sirt2 locates in the cytosol [39]. Sirts have various functions in protein modification, including ADP-ribosyltransferase activity and removing fatty-acyl groups from lysine residues. They are involved in energy metabolism, inflammation, genome stability, and aging [39].

The function of the class IV HDAC, HDAC-11, remains unclear. A few studies have suggested that this HDAC has a role in evolutionary conservation and the balance between immune activation and tolerance [40].

\section{Roles of HDACs in vascular function}

HDACs have been found to play important roles in vascular biology [27]. The crucial vascular functions of HDACs have been elucidated in knockdown studies. Class I HDACs (HDAC-1, -2 , and -3) are related to cardiac morphogenesis and endothelial survival. Montgomery et al. [41] demonstrated that HDAC-1 and -2 modulate cardiac morphogenesis, growth, and contractility. Cardiac-specific knockout of either HDAC1 or HDAC2 does not have significant effects on the cardiac phenotype. Cardiac-specific deletion of both HDAC-1 and HDAC-2 induces neonatal lethality and cardiac abnormalities, including cardiac arrhythmias and dilated cardiomyopathy. Zampetaki et al. [42] found that lentivirus-mediated HDAC-3 silencing in mice induces disruption of the basement membrane and rupture of blood vessels, resulting in a lethal phenotype. Class II HDACs (HDAC-5, - 7, and -9) are also associated with cardiovascular function. Chang et al. [43] demonstrated that knockout of HDAC-5 or -9 in mice results in cardiac hypertrophy. They also found that inhibition of HDAC-7 in mice induces the loss of EC-cell interactions and the rupture of blood vessels, resulting in embryonic lethality [44]. Class III HDACs have been shown to have protective functions in blood vessels, preventing atherosclerosis [45]. These studies have suggested that HDACs play vital roles in cardiovascular development and function.

\section{Roles of HDACs in shear-modulated EC function versus dysfunction}

Several studies have demonstrated that HDACs play important roles in modulating hemodynamics-induced EC function and dysfunction (Table 1). Studies in the laboratories of Shyy and Xu first demonstrated the roles of HDACs in flow-regulated EC function. Shyy and colleagues [46] found that PS induces HDAC-1 activation, resulting in the deacetylation of p53 and enhancement of p21 expression. Additionally, $\mathrm{Xu}$ and colleagues [47] demonstrated that HDAC3 is an important factor regulating PS-induced cell differentiation from stem cells into ECs through the Flk-1/phosphatidylinositol 3-kinase/Akt/HDAC3/p53/p21 pathway. In our previous studies, we identified the roles of class I and class II HDACs in modulating cellular functions, including proliferation, oxidation, and inflammation, in ECs in response to proatherogenic OS and atheroprotective PS (Fig. 3) [48]. We found that pro-atherogenic OS can induce the expression and nuclear accumulation of both class I (HDAC-1, - 2, and - 3) and class II HDACs (HDAC-5 and -7) in ECs. OS also can enhance the phosphorylation of class I HDACs to increase their activity. Krüppel like factor 2 (KLF-2) and NF-E2-related factor 2 (Nrf2), two important transcription factors that direct anti-inflammatory and antioxidant responses, govern approximately $70 \%$ shear-responsive genes [49]. OS-induced HDAC-1, -2 , and -3 can associate with Nrf2 to repress its binding to antioxidant response element to inhibit the expression of antioxidant NQO1 [48]. In addition, OS-induced HDAC-3 can cooperate with HADC-5 and -7 (class II HADCs) to associate with MEF2 and abolish the expression of anti-inflammatory KLF-2. Moreover, OS-induced HDAC-1, -2 , and -3 can modulate cell cycle regulators, i.e., upregulation of cyclin $\mathrm{A}$ and downregulation of $\mathrm{p} 21_{\mathrm{CIP} 1}$, to promote $\mathrm{EC}$ proliferation. In contrast, atheroprotective PS has no effect on inducing the expression and nuclear accumulation of both class I (HDAC-1, -2 , and -3 ) and class II (HDAC-5 and -7) HDACs, but induces phosphorylation-dependent nuclear export of class II HDACs. These PS-induced effects decrease HDAC levels in the nucleus to inhibit their repressive effects on transcription factors (Fig. 3) [48].

In vivo OS conditions created by the U-clip stenosed model and native circulation in rats were used to confirm that both class I and class II HDACs are highly expressed in ECs in response to OS. In addition, EC proliferation is found in the OS region of the rat U-clip stenosed model in vivo. Administration of the class I inhibitor VPA in experimental rats abolishes OS-induced EC proliferation in vivo. Our studies showed that hemodynamic forces, i.e., pro-atherogenic OS and atheroprotective PS, modulate the expressions or nuclear/ cytosolic shuttling of class I (HDAC-1, -2 , and - 3) and class II (HDAC-5 and -7) HDACs to regulate anti-inflammatory and antioxidant signaling by altering the acetylation of transcription factors, including MEF2 and $\mathrm{Nrf-2}$, in EC nuclei, which affect their transcription activities and the expression of downstream anti-inflammatory KLF-2 and antioxidant NQO1 genes. Moreover, pro-atherogenic OS-induced class I HDACs are involved in modulating EC proliferative signaling through regulation of cell cycle-related proteins, including cyclin $\mathrm{A}$ and p21 (Fig. 3) [48].

Other studies have also elucidated the roles of class I, class II, and class III HDACs in hemodynamics-modulated EC function and dysfunction (Table 1). Zampetaki et al. [42] found that orbital shaker-generated OS can induce 
Table 1 Roles of HDACs in hemodynamics-modulated endothelial function and dysfunction

\begin{tabular}{|c|c|c|c|c|c|c|}
\hline & HDACs & Class & Localization & Affected molecule & EC dysfunction/function & $\mathrm{Re}$ \\
\hline \multirow[t]{10}{*}{ Pro-atherogenic OS } & $\mathrm{HDAC}-1 \uparrow$ & I & Nucleus $\uparrow$ & Nrf2 (deacetylation)/NQO1 $\downarrow$ & Oxidation & 48 \\
\hline & & & & CyclinA $\uparrow / p 21 \downarrow$ & Proliferation & 48 \\
\hline & $\mathrm{HDAC}-2 \uparrow$ & I & Nucleus $\uparrow$ & Nrf2 (deacetylation)/NQO1 $\downarrow$ & Oxidation & 48 \\
\hline & & & & CyclinA $\uparrow / p 21 \downarrow$ & Proliferation & 48 \\
\hline & HDAC-3个 & 1 & Nucleus $\uparrow$ & Nrf2 (deacetylation)/NQO1 $\downarrow$ & Oxidation & 48 \\
\hline & & & & CyclinA $\uparrow / p 21 \downarrow$ & Proliferation & 48 \\
\hline & & & & AKT (phosphorylation) & Survival & 42 \\
\hline & 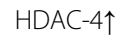 & $\|$ & Nucleus $\uparrow$ & MEF2 (deacetylation)/KLF2 $\uparrow$ VCAM $\downarrow$ & Inflammation & 48 \\
\hline & HDAC-5个 & $\|$ & Nucleus $\uparrow$ & MEF2 (deacetylation)/KLF2 $\downarrow$ VCAM $\uparrow$ & Inflammation & 48 \\
\hline & 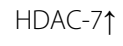 & $\|$ & Nucleus $\uparrow$ & MEF2 (deacetylation)/KLF2 $\uparrow$ VCAM $\downarrow$ & Inflammation & 48 \\
\hline \multirow[t]{8}{*}{ Atheroprotective PS } & HDAC-1 & I & Nucleus/Cytosol & p53 (deacetylation)/p21 & Antiproliferation & 46 \\
\hline & HDAC-3 & I & Nucleus/Cytosol & MEF2 (acetylation)/KLF2 $\uparrow$ VCAM $\downarrow$ & Anti-inflammation & 48 \\
\hline & & & $?$ & p53 (deacetylation)/p21 & Differentiation into ECs & 47 \\
\hline & HDAC-5 & $\|$ & Cytosol $\uparrow$ & MEF2 (acetylation)/KLF2 $\uparrow$ VCAM $\downarrow$ & Anti-inflammation & 48 \\
\hline & & & & MEF2 (acetylation)/KLF2 $\uparrow$ eNOS $\uparrow$ & NO production & 50 \\
\hline & 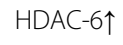 & $\|$ & Cytosol $\uparrow$ & a-tubulin & Migration & 51 \\
\hline & HDAC-7 & $\|$ & Cytosol $\uparrow$ & MEF2 (acetylation)/KLF2 $\uparrow$ VCAM $\downarrow$ & Anti-inflammation & 48 \\
\hline & Sirt $1 \uparrow$ & III & $?$ & eNOS (deacetylation) & NO production & 52 \\
\hline
\end{tabular}

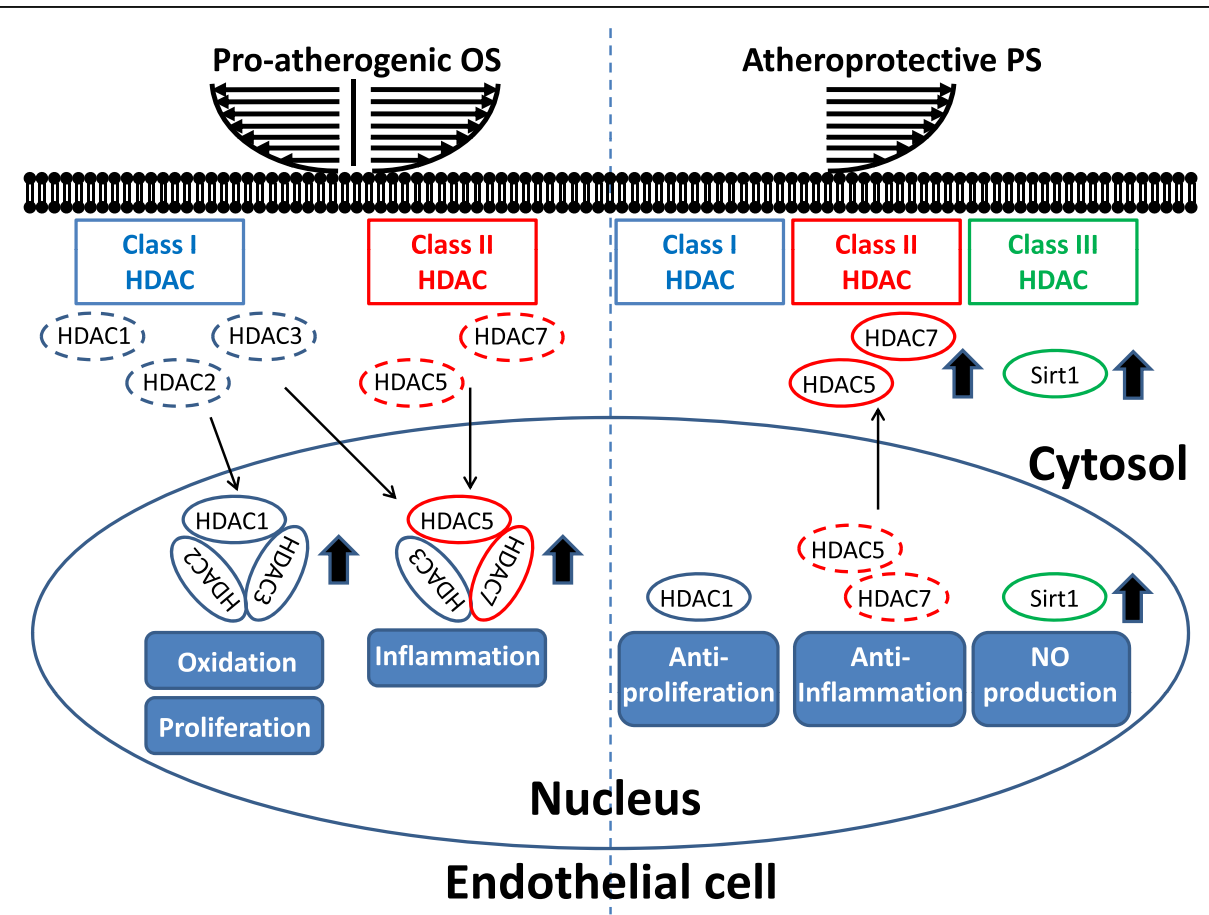

Fig. 3 Roles of HDACs in modulating hemodynamics-regulated EC dysfunctions, including proliferation, inflammation, and oxidation. Pro-atherogenic OS induces expression and nuclear accumulation of both class I (HDAC-1, -2 , and -3) and class II HDACs (HDAC-5 and -7). Moreover, OS further enhances the formation of HDAC-1/HDAC-2/HDAC-3 and HDAC-3/HDAC-5/HDAC-7 heterocomplexes to promote proliferation, inflammation, and oxidation. In contrast, atheroprotective PS induces phosphorylation-dependent nuclear export of class II HDACs to decrease HDAC levels in the nucleus to inhibit their effects on proliferation, inflammation, and oxidation. On the other hand, PS induces the expression of Class III (Sirt1) to enhance NO production 
serine/threonine phosphorylation of HDAC-3 to modulate endothelial survival and integrity via AKT activation. Additionally, Wang et al. [50] found that PS can induce phosphorylation-dependent nuclear export of HDAC-5 in ECs through a calcium/calmodulin-dependent pathway. PS-induced nuclear export of HDAC-5 decreases the ability of HDAC-5/MEF2 to enhance MEF2 acetylation and transcriptional activity and induce the expressions of KLF-2 and eNOS, which are key mediators involved in flow-mediated anti-inflammatory and -protective functions. Wang et al. [51] indicated that PS increases the expression of HDAC-6 to deacetylate tubulin and induce EC migration. Class III HDACs (e.g., Sirt1) have also been shown to have roles in modulating shear-dependent EC function. For example, Chen et al. [52] indicated that PS increases the expression and activity of Sirt1. PS-induced Sirt1 can associate with eNOS to induce eNOS deacetylation. These results suggested that PS-induced Sirt1 (a class III HDAC) increases NO bioavailability in ECs.

In addition to hemodynamics-modulated EC function and dysfunction, HDACs are also involved in the progression of vascular disorders, including atherosclerosis $[53,54]$. HDACs are involved in various processes in atherosclerotic formation, including blood glucose and plasma lipid elevation, monocyte accumulation and migration, foam cell formation, vascular smooth muscle cell (SMC) phenotype switch, fibrous cap formation, plaque disruption, and thrombosis [54]. Kee et al. [55] showed that the HDAC inhibitor trichostatin A activates KLF-4 to inhibit balloon injury-induced neointimal hyperplasia. Other studies have also linked different classes of HDACs to vascular disorders, including atherosclerosis. For example, Findeisen et al. [56] showed that endovascular injury of the mouse femoral artery induces the expression of class I HDACs and the formation of neointima. Treatment with the HDAC inhibitor scriptaid inhibits injury-induced neointima formation. However, knockdown of HDAC3 induces EC apoptosis, as shown by increased extensive membrane blebs and cytosolic nucleosomes and enhanced annexin V staining, thereby accelerating neointima formation [42]. Usui et al. [57] found that the class IIa HDAC inhibitor MC1568 inhibits not only the activation of HDAC4 in the neointima region but also the formation of neointimal hyperplasia in a mouse carotid ligation model. Class III HDACs have been shown to prevent atherosclerosis in blood vessels $[45,58,59]$. Overexpression of Sirt1 in the endothelium in ApoE-deficient $\left(\mathrm{ApoE}^{-/-}\right)$mice induces the expression of eNOS, represses the expression of adhesion molecules, and subsequently inhibits the progression of atherosclerosis [58]. Haploinsufficiency of Sirt6 in $\mathrm{ApoE}^{-/-}$mice has been found to promote atherogenesis [59]. These studies indicate that HDACs play vital roles in the progression of vascular disorders, including atherosclerosis.

\section{Non-coding RNAs in hemodynamics-directed EC function versus dysfunction The biosynthesis and function of microRNAs (miRs)}

MiRs, which are small noncoding RNAs (18-22 nucleotides in length), have emerged as new post-transcriptional repressors that function by binding to the mRNA of target genes to initiate their degradation and translational repression [28-30]. The transcriptional expression of miRs is regulated by transcription factors, including retinoic acid receptor (RAR) and KLF-2 [30, 60, 61]. These transcription factors show variations in acetylation or expression, affecting their binding activity for promoters or enhancers of miRs to modulate miR transcription in ECs. Subsequently, miRs are transcribed from DNA to generate primary miRs (pri-miRs) in the nucleus by RNA polymerase II or III. Pri-miRs are processed by a processor complex composed of DGCR8 and Drosha into 60-100nucleotide precursor miRs (pre-miRs) with a 3' overhang hairpin structure. Pre-miRs are then transported into the cytoplasm by exportin-5. In cytosol, pre-miRs are processed by the RNA-induced silencing complex, which contains Ago2 and Dicer, to remove the hairpin structure to form a 22 -nucleotide $\mathrm{miR} / \mathrm{miR}$ * duplex. The $\mathrm{miR}$ strand of the $\mathrm{miR} / \mathrm{miR}^{*}$ duplex is processed into mature $\mathrm{miR}$, whereas the miR* strand of $\mathrm{miR} / \mathrm{miR}^{*}$ is degraded. Mature miR further cooperates with Dicer and other associated proteins to form an miR-induced silencing complex and base pairs with 6-8 nucleotides within the 3 '-untranslated region (UTR) of target genes, which exerts important functions in modulating target genes, including mRNA degradation or translation repression [28-30].

\section{Roles of miRs in vascular function}

The effects of miRs on the regulation of gene expression are involved in various physiological functions in health and disease [28-30]. miRs have been found to play important roles in various organs and tissues, including heart, muscle, liver, and kidney [62]. Blockage of miR biosynthesis in zebrafish and mice by suppression of important miR processers, including Dicer, has demonstrated that miRs modulate cardiovascular functions. Dicer-null zebrafish embryos show disrupted blood circulation and severe defects in cardiac development [63]. Dicer-deficient mice, generated by homologous recombination in embryonic stem cells, also show severe defects in blood vessel formation and maintenance [64]. Cardiac-specific deletion of Dicer modulates miR expression to induce dysregulation of adhesion proteins, cardiac remodeling, and heart failure $[65,66]$. In addition, several miRs have been found to regulate cardiovascular function. For example, miR-1 prevents highfat diet-induced endothelial permeability in $\mathrm{ApoE}^{-/}$ mice [67]. In contrast, knockout of miR-133a in mice results in dilated cardiomyopathy and heart failure [68]. 
Knockdown of miR-126 in zebrafish induces the loss of vascular integrity and promotes hemorrhage during embryogenesis [69]. Similarly, deletion of miR-126 in mice results in severe systemic edema, multifocal hemorrhage, and ruptured blood vessels throughout embryogenesis [70].

\section{Transcriptional regulation of miRs by hemodynamic forces in ECs}

Hemodynamic forces, i.e., OS and PS, exert differential effects on modulating miR expression and function in ECs $[29,30]$. Chien and collaborators [71, 72] first used an miR microarray to examine the expression profiles of miRs in ECs in response to atheroprotective PS in vitro. Eight upregulated miRs and thirteen downregulated miRs have been found in ECs in response to PS. Among these shear-regulated miRs, $m i R-19 a$ and $m i R-23 b$ have been found to be robustly upregulated by PS to modulate EC growth arrest [71, 72]. In addition, $\mathrm{Ni}$ et al. [73] also used an miR microarray to compare miR profiles in ECs subjected to OS versus PS in vitro; they showed that $m i R-663$ was the miR with the highest expression in OS-treated ECs. OS-induced miR-663 activates pro-inflammatory responses in ECs. Davies and colleagues [74] used an miR microarray to compare the expression profiles of miRs in the endothelium of atherosusceptible regions versus atheroprotected regions in normal adult swine in vivo. Among $1139 \mathrm{miRs}$, they found seven downregulated miRs and twenty seven upregulated miRs in the endothelium of atherosusceptible regions (OS regions) in comparison to atheroprotected regions (PS regions). Additionally, $m i R-10 a$ was identified as the miR with the lowest expression in the endothelium of atherosusceptible regions (OS regions) versus atheroprotective regions (PS regions). They also demonstrated that knockout of miR-10a activates IkB/NF-kB-mediated proinflammatory responses in ECs in vitro. Moreover, Son et al. [75] used an miR microarray to identify the miR profiles in ECs subjected to OS versus PS in vivo using a partially ligated mouse model. They further used in vitro flow conditions to confirm the in vivo results and found that miR-712 was the most robustly upregulated miR in ECs in response to OS in both in vivo and in vitro. These studies suggested that the expression of miRs is differentially modulated by different hemodynamic forces, including pro-atherogenic OS and atheroprotective PS, to affect EC function.

Transcriptional initiation, which is regulated by transcription factors, is important for modulating miR expression in ECs in response to hemodynamic forces. Hemodynamic forces, i.e., OS and PS, modulate the activity and expression of transcription factors, i.e., RAR $\alpha$ and KLF-2, to regulate miR expression [30, 60, 61]. Our previous study identified the mechanisms through which hemodynamic forces modulate transcriptional activity of RAR $\alpha$, resulting in changes in the expression of miR-10a and affecting anti-inflammatory signaling and cellular functions in ECs (Fig. 4) [60,76]. Our results in an in vitro flow system showed that atheroprotective PS induces the expression, nuclear accumulation, and association of RAR $\alpha$ and retinoid X receptor (RXR) $\alpha$ (an enhancer of RAR $\alpha$ ) to promote the binding of RAR $\alpha$ to RA-responsive elements in the enhancer region of miR$10 a$, resulting in increased miR-10a expression in ECs. PS-induced miR-10a further targets the 3 '-UTR of proinflammatory GATA6 to repress its expression, leading to inhibition of VCAM-1. In contrast, pro-atherogenic OS induces the association of RAR $\alpha$ with HDAC-3/-5/ - 7 (repressors of RAR $\alpha$ ) to repress RAR $\alpha$-directed miR$10 a$ signaling. These in vitro results were further confirmed in vivo by en face staining of aortic arch (OS region) versus the straight thoracic aorta (PS region) in rats. Likewise, the transcription factor KLF-2 is regulated by hemodynamic forces to modulate the expression of miRs. KLF-2 is a shear-sensitive transcription factor whose expression is upregulated by atheroprotective PS, but downregulated by pro-atherogenic OS in vivo and in vitro [77]. Nicoli et al. [61] demonstrated that KLF-2 is induced by PS to modulate miR-126 and stimulate angiogenesis in zebrafish. The transcriptional start site of miR-126 is predicted to contain a KLF-2 binding site in humans. Moreover, Chien and colleagues showed that KLF-2 also modulates PS-induced miR-23b [78]. In addition to miR-126 and miR-23b, KLF-2 is also predicted to transcriptionally regulate several miRs, including $m i R-10 a, m i R-30 a$, miR-143, miR-145, and miR-150 [30]. These studies have suggested that the expression or acetylation of transcription factors, e.g., RAR $\alpha$ or KLF-2, can be differentially regulated by pro-atherogenic OS and atheroprotective PS to modulate the transcriptional expression of miRs.

\section{Roles of miRs in shear-modulated EC function versus dysfunction}

MiRs have also been shown to play essential roles in modulating hemodynamics-induced EC functions, including proliferation, inflammation, and oxidation. One group of miRs, including miR-19a, miR-23b, miR-101, $m i R-155$, and miR-126-5p is involved in hemodynamicsmodulated EC proliferation (Table 2) $[29,30]$. As described above, miR-19a and miR-23b are upregulated by PS to modulate EC growth arrest by degrading cell cycle regulatory proteins, including cyclin D1 and E2F1 [71, 72]. PS induces KLF-2-dependent biosynthesis and expression of $m i R-23 b$, leading to cyclin-dependent kinase-activating kinase complex repression and cell cycle suppression [78]. Chen et al. [79] found that $m i R-101$ is upregulated by PS to target proliferative signaling 


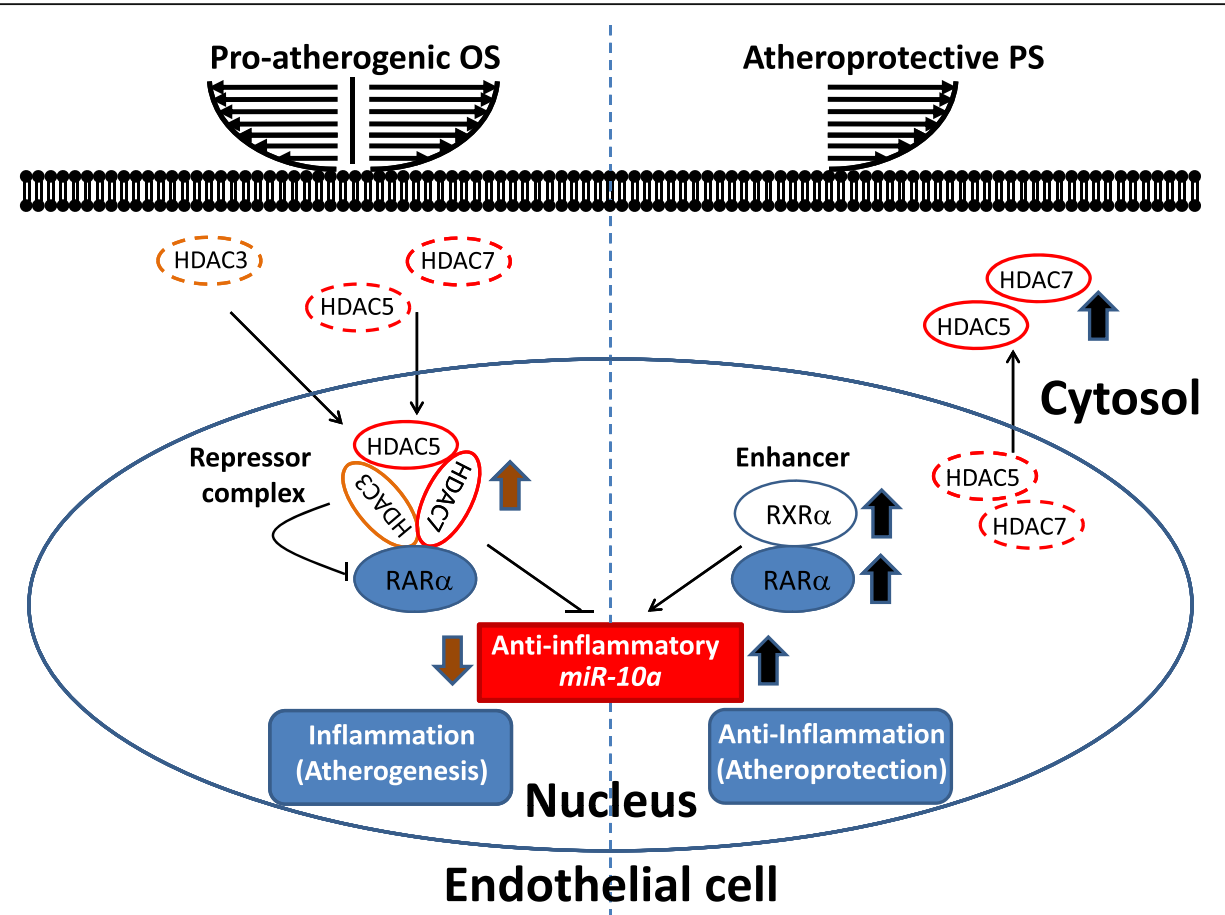

Fig. 4 Schematic diagram of regulatory machinery for modulating atherosclerosis. Atheroprotective PS induces the expression, nuclear accumulation, and association of RARa (director) and RXRa (enhancer) to promote RARa/RARE binding and miR-10a expression in ECs. PS-induced miR-10a targets pro-inflammatory transcription factor GATA6 to repress its expression to inhibit downstream VCAM-1 expression. PS-induced RARa/miR-10a signaling elicits anti-inflammatory signaling in ECs. In contrast, pro-atherogenic OS induces the association of RARa with HDAC-3/-5/- 7 (repressors) to repress RARa-directed miR-10a signaling to induce pro-inflammatory responses in ECs

molecules, including mTOR, to repress its expression and induce cell arrest in ECs. Weber et al. [80] demonstrated that PS induces miR-155 to inhibit EC proliferation and migration. In addition, Schober et al. [81] showed that pro-atherogenic OS inhibits miR$126-5 p$ to induce the expression of its direct target Dlk1, resulting in enhanced EC proliferation and promotion of atherosclerosis.

Another group of miRs, including miR-10a, miR-92a, miR-633, miR-712, miR-21, miR-34a, miR-146a, miR708, miR-451, and miR-98, are involved in shear-modulated EC inflammation (Table 2) [29, 30]. As described above, knockdown of miR-10a in ECs activates NF-kB signaling [74]. Moreover, miR-10a can be differentially regulated by hemodynamic forces, including PS and OS, to modulate pro-inflammatory GATA6/VCAM-1 via the association of RAR $\alpha$ with RXR $\alpha$ and HDACs [60, 76]. $\mathrm{Wu}$ et al. [82] demonstrated that miR-92a regulates hemodynamics-dependent EC inflammation and function. Moreover, PS downregulates, whereas OS upregulates, miR-92a expression to modulate KLF-2 expression in ECs. Shear-modulated miR-92a can further regulate the expression of eNOS and thrombomodulin (TM) to affect EC function. Ni et al. [73] showed that miR-663 can be induced by pro-atherogenic OS to activate proinflammatory responses in ECs. Son et al. [75] found that $m i R-712$ is upregulated by OS to inhibit tissue inhibitor of metalloproteinase 3 , enhance the release of soluble tumor necrosis factor- $\alpha$ and the adhesion of monocytes, and activate pro-inflammatory responses in ECs. They further demonstrated that treatment with a miR-712 antagonist inhibits the progression of atherosclerosis. Zhou et al. [83] found that $m i R-21$ can be induced by OS to bind to the $3^{\prime}$-UTR of peroxisome proliferator-activated receptor (PPAR) $\alpha$ for degradation. OS-induced PPAR $\alpha$ degradation decreases its inhibitory effect on the transcription factor activator protein-1 and enhances the expression of downstream VCAM-1 and MCP-1 to activate pro-inflammatory responses in ECs. Fan et al. [84] found that $m i R-34 a$ is upregulated by OS, but downregulated by PS. Moreover, OS-induced miR$34 a$ enhances the expression of ICAM-1 and VCAM-1 through NF- $\mathrm{KB}$ signaling, consequently promoting monocyte adhesion to ECs. Our recent study demonstrated that PS promotes the expression of anti-inflammatory miRs, including miR-146a, miR-708, miR-451, and miR-98. PS-induced miR-146a, miR-708, miR-451, and miR-98 directly target interleukin-1 receptor-associated kinase, inhibitor of NF- $\mathrm{kB}$ kinase subunit- $\gamma$, interleukin-6 receptor, and conserved helix-loop-helix ubiquitous kinase genes, respectively, to inhibit NF-kB signaling [85]. Another group of miRs, including miR- 
Table 2 Roles of non-coding RNAs in hemodynamics-modulated endothelial function and dysfunction

\begin{tabular}{|c|c|c|c|c|c|c|}
\hline & $\begin{array}{l}\text { Non-coding } \\
\text { RNA }\end{array}$ & Classification & Direct target & Affected molecule & EC dysfunction/function & Ref \\
\hline \multirow[t]{14}{*}{ Pro-atherogenic OS } & $\operatorname{miR}$ & & & & & \\
\hline & $\operatorname{miR}-126-5 p \downarrow$ & Antiproliferative miR & Dlk1 $\uparrow$ & & Proliferation & 81 \\
\hline & miR-10a $\downarrow$ & Anti-inflamatory miR & GATA6个 & VCAM-1个 & Inflammation & 60,76 \\
\hline & & & TAK1, $\beta$ TRC $\uparrow$ & NF-kB p65 个 & Inflammation & 74 \\
\hline & & & & E-sel, VCAM-1, $\uparrow$ & Inflammation & 74 \\
\hline & & & & IL-6, IL-8, MCP-1个 & Inflammation & 74 \\
\hline & $\operatorname{miR}-92 a \uparrow$ & Inflammatory miR & $\mathrm{KLF} 2 \downarrow$ & IL-6, MCP-1 $\uparrow, T M \downarrow$ & Inflammation & 82 \\
\hline & $\operatorname{miR}-663 \uparrow$ & Inflammatory miR & $?$ & KLF4 $\downarrow$ & Inflammation & 73 \\
\hline & $\operatorname{miR}-712 \uparrow$ & Inflammatory miR & $\downarrow T I M P$ & 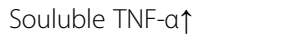 & Inflammation & 75 \\
\hline & $\operatorname{miR}-21 \uparrow$ & Inflammatory miR & $\downarrow P P A R a$ & 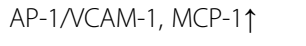 & Inflammation & 83 \\
\hline & $\operatorname{miR}-34 a \uparrow$ & Inflammatory miR & $?$ & NF-KB/NCAM-1, ICAM-1 $\uparrow$ & Inflammation & 84 \\
\hline & $\operatorname{miR}-21 \uparrow$ & Oxidative miR & SOD $\downarrow$ & $?$ & Oxidation & 30 \\
\hline & $\operatorname{miR} 17^{*} \uparrow$ & Oxidative miR? & SOD, GPx, Trx2 $\downarrow$ & $?$ & Oxidation? & 30 \\
\hline & $\operatorname{miR}-92 a \uparrow$ & NO-related miR & KLF2 $\downarrow$ & eNOS $\downarrow$ & NO repression & 82 \\
\hline \multirow[t]{22}{*}{ Atheroprotective PS } & $\operatorname{miR}$ & & & & & \\
\hline & $\operatorname{miR}-19 a \uparrow$ & Anti-proliferative miR & CyclinA $\downarrow$ & $?$ & Antiproliferation & 71 \\
\hline & $\operatorname{miR}-23 b \uparrow$ & Anti-proliferative miR & $E 2 F \downarrow$ & Rb phosphorylation $\downarrow$ & Antiproliferation & 72 \\
\hline & & & $?$ & CAK complex (repression) & Antiproliferation & 78 \\
\hline & $\operatorname{miR}-101 \uparrow$ & Anti-proliferative miR & mTOR $\downarrow$ & $?$ & Antiproliferation & 79 \\
\hline & $\operatorname{miR}-155 \uparrow$ & Anti-proliferative miR & 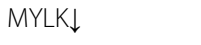 & RhoA & Antiproliferation Anti-proliferation & 80 \\
\hline & $\operatorname{miR}-10 a \uparrow$ & Anti-inflamatory miR & GATA6 $\downarrow$ & VCAM-1 $\downarrow$ & Anti-inflammation & 60,76 \\
\hline & $\operatorname{miR}-146 a \uparrow$ & Anti-inflammatory miR & IRAK $\downarrow$ & NF-KB signaling $\downarrow$ & Anti-inflammation & 85 \\
\hline & $\operatorname{miR}-708 \uparrow$ & Anti-inflammatory miR & $\mid K K-\gamma \downarrow$ & NF-KB signaling $\downarrow$ & Anti-inflammation & 85 \\
\hline & $\operatorname{miR}-451 \uparrow$ & Anti-inflammatory miR & $\mid \mathrm{L}-6 \mathrm{R} \downarrow$ & NF-KB signaling $\downarrow$ & Anti-inflammation & 85 \\
\hline & $\operatorname{miR}-98 \uparrow$ & Anti-inflammatory miR & $\mathrm{CHUK} \downarrow$ & NF-KB signaling $\downarrow$ & Anti-inflammation & 85 \\
\hline & 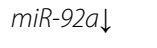 & Anti-inflammatory miR & KLF2 $\uparrow$ & $\mathrm{TM} \uparrow$ & Anti-inflammation & 82 \\
\hline & & NO-related miR & $\mathrm{KLF} 2 \uparrow$ & eNOS $\uparrow$ & NO production & 82 \\
\hline & $\operatorname{miR}-21 \uparrow$ & NO-related miR & PTEN $\downarrow$ & eNOS (phosphorylation)^ & NO production & 87 \\
\hline & IncRNAs & & & & & \\
\hline & STEEL $\downarrow$ & Turnover-related InRNA & & eNOS, KLF-2^ & Turnover, migration & 93 \\
\hline & & & & & NO production??? & \\
\hline & MANTIS $\uparrow$ & Aligment-related InRNA & & Smad6, Sox18 & Cell alignment & 94 \\
\hline & & & & & Angiogenesis & \\
\hline & LASSIE $\uparrow$ & Aligment-related InRNA & & & Cell alignment & 95 \\
\hline & & & & & Cell-cell interaction & \\
\hline & 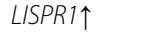 & Migration-related InRNA & & S1PR1 signaling $\uparrow$ & Migration & 96 \\
\hline
\end{tabular}

$21, m i R-17 *$, and $m i R-30 b$, have been shown to regulate shear-dependent oxidative responses in ECs by mediating pro-oxidant or antioxidant enzymes, including SOD, GPx, thioredoxin-dependent peroxidase, and catalase (Table 2) $[29,30]$. OS has been shown to induce the expression of $m i R-21$, which targets the antioxidant SOD [30]. In addition, PS has been shown to inhibit the expression of $m i R-17$, whose product $m i R-17^{*}$ is proposed to target antioxidant SOD, GPx, and Trx2 [30]. Thus, $m i R-21$ and $m i R-17^{*}$ may be involved in shear-modulated expression of antioxidant enzymes. The balance between NO and ROS is vital for maintaining EC function [86]. MiR-21 and miR-92a have been found to be regulated by hemodynamic forces to modulate NO production. Weber et al. [87] demonstrated that miR-21 is induced by PS, resulting in the phosphorylation of eNOS 
and the enhancement of $\mathrm{NO}$ production. Wu et al. [82] demonstrated that inhibition of $m i R-92$ by PS enhances eNOS expression and promotes NO production, whereas induction of $m i R-92$ by OS inhibits eNOS-directed NO production.

In addition to hemodynamics-modulated EC dysfunction, several miRs have been shown to be related to atherosclerosis. In our recent studies in $\mathrm{ApoE}^{-/-}$mice, decreases in endothelial and serum miR-10a were found to be related to atherogenesis. Treatment of $\mathrm{ApoE}^{-/-}$ mice with pre-miR-10a or RAR $\alpha / R X R \alpha$ agonists can rescue miR-10a expression to inhibit the formation of atherosclerosis [76]. Schober et al. [81] showed that miR126-5p suppresses the expression Dlk1 to prevent the progression of atherosclerosis. Loyer et al. [88] demonstrated that in vivo knockdown of miR-92a in $\mathrm{LDLR}^{-1-}$ mice inhibits endothelial inflammation and atherosclerosis progression. Moreover, Son et al. [75] demonstrated that treatment of $\mathrm{ApoE}^{-1-}$ mice with a miR-712 antagonist inhibits the progression of atherosclerosis. Our recent results showed that treatment of mice with lentivirus carrying mature miR-146a blocks neointima formation in a mouse carotid artery ligation model [85].

\section{Other non-coding RNAs relevant to shear-regulated EC dysfunction and atherosclerosis}

In addition to miRs, recent studies indicate that other non-coding RNA categories, i.e., long noncoding RNAs (lncRNAs) and circular RNAs (circRNAs), also play important roles in the epigenetic regulation of endothelial function and atherosclerotic vascular diseases [89-92]. LncRNAs are non-coding RNAs with more than $200 \mathrm{nu}-$ cleotides. They modulate various cellular processes in the nucleus and cytoplasm. In the nucleus, lncRNAs serve as histone modulators, enhancers or repressors, chromatin remodeling modulators, or transcription factor regulators to modulate transcription. In the cytoplasm, lncRNAs modulate mRNA stability, translation, and protein scaffold $[89,90]$. Moreover, they can regulate protein phosphorylation and signaling transduction $[89,90]$. On the other hand, circRNAs are circular non-coding RNAs, which are different from linear miRNA and lncRNAs. CircRNAs typically link the $5^{\prime}$ end to the $3^{\prime}$ end of RNAs to generate circular structure. They are also located in nucleus and cytoplasm to regulate cellular function. The function of circRNAs has been identified to regulate gene expression through transcriptional, post-transcriptional, and translational modulations [91, 92].

Spliced-transcript endothelial-enriched lncRNA (STEEL) and MALAT1 were first identified to be shear-sensitive lncRNAs by Man et al. and Leisegang et al. (Table 2) $[93,94]$. STEEL is an EC-specific lncRNA that enhances cell turnover and migration and has ability to promote blood vessel formation and maturation. Moreover, STEEL can transcriptionally regulate the expression of eNOS and KLF2, which are two major mediators of shear-responsive responses. STEEL performs epigenetic modulation in the transcriptional changes, including increased chromatin accessibility and histone methylation at the eNOS and KLF2 promoters. The RNA and IncRNA of STEEL are decreased in ECs in response to atheroprotective PS, eliciting the upregulation of both KLF2 and eNOS. Overexpression or knockdown of KLF2 in ECs demonstrated that KLF2 has feedback inhibitiory effects on STEEL under atheroprotective PS condition [93]. MANTIS is an additional important lncRNA that facilitates endothelial angiogenic function. The level of MANTIS is enhanced by atheroprotective flow. Knockdown assay demonstrated that MANTIS can modulate shear-induced EC alignment and angiogenic sprounding [94]. On the other hand, Stanicek et al. [95] used RNA sequencing to identify that LASSIE is an atheroprotective flow-induced lncRNA. The shear-induced LASSIE is modulated by KLF2. Knockdown of LASSIE in ECs showed that LASSIE plays vital roles in cell-cell interactions and atheroprotective PS-induced EC alignment (Table 2). In addition, Josipovic et al. [96] found that long intergenic noncoding RNA antisense to S1PR1 (LISPR1) is also an atheroprotective flow-induced lncRNA. Its function has been found to regulate S1PR1 expression and S1P signaling pathway (Table 2). Moreover, several lncRNAs, including H19 [97-99], SENCR [100], MEG3 [101], and RNCR3 [102], are related to atherosclerosis. H19 expression has been found to be higher in human atherosclerotic lesion [103]. Moreover, high level of H19 is also found in $\mathrm{ApoE}^{-/-}$mice [98]. Overexpression of $H 19$ in ECs can induce upregulation of p38 MAPK and NF-kB and cellular proliferation [97]. In addition, the level of $H 19$ is higher in the plasma from human CAD patients with heart failure, as compared with that with normal cardiac function [99]. SENCR is a vascular lncRNA, which is enriched in ECs and SMCs. The expression of SENCR in ECs is identified to be downregulated in human CAD patients compared to healthy subjects [100]. Loss- and gain-of-function studies in ECs demonstrated that SENCR can modulate proliferation, migration, and tube formation of ECs. Moreover, SENCR has also been found to regulate the expression of pro-angiogenic genes, i.e., CCL5, CEACAM1 and CX3CL1. $M E G 3$ is a lncRNA that is downregulated in human CAD tissues and proliferative ECs. MEG3 has been identified to suppress EC proliferation through inhibiting the expression of cyclin D1, ki-67 and PCNA. In addition, MEG3 also can inhibit the expressions of type I collagen, type $\mathrm{V}$ collagen and proteoglycan [101]. RNCR3 is a lncRNA which is expressed in ECs and SMCs to regulate their proliferation, migration, and apoptosis. The expression of $R N C R 3$ has been found to be highly expressed in the atherosclerotic aortas of $\mathrm{ApoE}^{-/-}$mice and human 
specimens. Knockdown of $R N C R 3$ in $\mathrm{ApoE}^{-/-}$mice promotes the formation and enhances the levels of total cholesterol, triglycerides, and pro-inflammatory factors in blood [102].

Study on CircRNAs is a new field in vascular biology. Until now, only a few literatures correlate circRNAs to EC function and atherosclerosis. Dang et al. [104] used circRNA microarray to identify the expression profiles of hypoxia-stimulated ECs. They found 14 downregulated and 22 upregulated circRNAs in hypoxia-stimulated ECs. Among these circRNAs, circ_0010729 was found to be significantly upregulated. Knockdown experiment of circ_0010729 demonstrated that circ_0010729 promotes EC proliferation and migration and inhibits EC apoptosis. On the other hand, Liu et al. [105] identified that cZNF609 was upregulated in high glucose- and hypoxia stress-treated ECs in vivo and in vitro. Knockdown and overexpression studies of $c Z N F 609$ demonstrated that cZNF609 can induce retinal vessel loss and pathological angiogenesis in vivo. Moreover, knockdown of cZNF609 in ECs demonstrated that cZNF609 can inhibit EC migration, tube formation, and protective effect against oxidative stress and hypoxia stress in vitro [105]. Holdt et al. [106] found that circular antisense non-coding RNA in the INK4 locus (circANRIL) impairs ribosome biogenesis and induces p53 activation to enhance apoptosis and reduce proliferation of SMCs and macrophage, and hence plays atheroprotective roles in vascular tissue. Overexpression of circANRIL in SMCs or macrophage can induce cell apoptosis and decrease cell proliferation. They also compared SMCs from different human CAD patients to demonstrate that high circANRIL expression induces apoptosis and reduces proliferation of SMCs. Song et al. [107] further elucidated the role of circANRIL in ECs in atherosclerotic rat model. Overexpression of circANRIL in atherosclerotic rat promotes apoptosis and inflammation of ECs and development of atherosclerotic plaques. Additionally, the levels of serum IL-1, IL-6, MMP-9 were increased in the circANRIL-expressed rats. They also suggested that circANRIL inhibition has a potential to be developed as therapeutic strategy for atherosclerosis treatment [107]. CircRNA in blood serum has also been proposed to be a diagnostic biomarker for CAD. Zhao et al. [108] used RNA microarray to compare peripheral blood circRNAs from 12 CAD patients and those from 12 healthy controls. They found 12 upregulated and 10 downregulated circRNAs in CAD patients. Among these circRNAs, they further identified hsa_circ_0124644 as a potential biomarker for CAD. Moreover, Pan et al. [109] used microarray analysis to identify 18 upregulated and 6 downregulated circRNAs in blood serum of CAD patients in comparison to healthy subjects.
DNMTs in hemodynamics-directed EC function versus dysfunction Classification and function of DNMTs

In DNA methylation, a methyl group is added to the fifth carbon of a cytosine to form 5-methylcytosine $(5 \mathrm{mC})$ [110]. The regulation of most promoters in human genes is related to $\mathrm{CpG}$ islands, which are located in or near the promoter region and maintained in an unmethylated state to promote gene transcription [110]. In contrast, these DNA regions can be methylated to recruit methyl-CpG binding proteins and activate repressive machinery or inhibit the binding of transcription factors to promoters, resulting in chromatin compaction and inhibiting gene transcription [111]. DNA methylation plays essential roles in embryonic development and biological functions. Dysregulation of DNA methylation, i.e., hyper- or hypomethylation, results in various diseases, including cardiovascular diseases [112, 113].

DNA methylation is mainly regulated by DNMTs, which catalyze the addition of a methyl group to cytosine. De novo methyltransferases preferentially bind to unmethylated DNA to induce DNA methylation, whereas maintenance methyltransferases binds to hemimethylated DNA to induce DNA methylation. There are several DNMT isoforms, including DNMT1, DNMT3a, and DNMT3b, to be found $[31,114,115]$. DNMT1 is the most abundant DNMT in adult cells and acts primarily as a maintenance methylase. It has also been shown to have de novo methyltransferase activity. Deletion of DNMT1 induces genome hypomethylation and results in embryonic lethality [116]. DNMT3a and - $3 \mathrm{~b}$ are classified as de novo methyltransferases. Deletion of both of DNMT3a and -3b results in early embryonic lethality. DNMT3a has been found to play roles in late development, whereas DNMT3b is involved in early development [117].

\section{Roles of DNA methylation and DNMTs in vascular function}

Aberrant DNA methylation and methyltransferase expressions are related to vascular disorders [118]. The reduction in genomic $5 \mathrm{mC}$ is observed in advanced atherosclerotic lesions in humans and $\mathrm{ApoE}^{-/-}$mice. Moreover, hypomethylation of $\mathrm{CpG}$ islands is also found in the arteries of patients with atherosclerosis in comparison to that in control arteries [119]. However, the atheroprotective gene encoding estrogen receptor $\beta$ is hypermethylated in atherosclerotic lesions of coronary arteries compared with that in normal control arteries [120]. In addition, the expressions of eNOS and vascular endotheilial growth factor receptor 2 (VEGF-R2) are repressed by methyl-CpG-binding domain protein 2 (MBD2), an MBD protein that binds to methylated DNA to mediate DNA methylation-dependent transcriptional repression, through directly binding to the methylated CpG elements in the promoters of these genes. 
Knockdown of MBD2 activates pro-angiogenic and protective signals, e.g., upregulation of VEGF-R2 and eNOS, to enhance EC angiogenesis and protect ECs against $\mathrm{H}_{2} \mathrm{O}_{2}$-induced apoptosis in vitro. Moreover, deletion of MBD2 in mice protects mice against hind-limb ischemia injury in vivo [121]. Thus, global DNA hypomethylation may be observed in atherosclerotic arteries, whereas specific DNA hypermethylation may occur in atheroprotective genes.

Notably, DNMTs are activated under pro-atherogenic conditions, i.e., a high-fat diet or high low-density lipoprotein (LDL) cholesterol levels. DNMT1 has been found to be overexpressed and activated in $\mathrm{ApoE}^{-/-}$ mice fed a high-fat diet [122]. Treatment of ECs with LDL cholesterol induces DNMT1 expression. In contrast, high levels of serum homocysteine, which acts as a source of methyl groups for methylation responses and has been shown to be a risk factor for EC inflammation and atherosclerosis, are found in patients with atherosclerosis [123]. Therefore, DNA methylation and DNMT play important roles in regulating vascular dysfunction.

\section{Effects of hemodynamic forces on modulating DNMTs in ECs}

Recent studies have shown that DNMTs, including DNMT1 and DNMT3a, are modulated by hemodynamic forces, i.e., OS and PS, to regulate inflammatory signaling (Table 3). Davies and coworkers [124] first showed that DNMT3a can be modulated by pro-atherogenic OS to regulate EC function. OS upregulates DNMT3a, which can bind to the promoter of KLF-4, a transcription factor that activates anti-inflammatory and antiproliferative responses in ECs, and induce DNA methylation of CpG islands in the KLF-4 promoter, resulting in repression of KLF-4 transcription. Shear-inhibition of KLF-4 further modulates downstream molecules, including NOS3, $\mathrm{TM}$, and MCP-1. These in vitro results were confirmed in vivo by observation of the hypermethylation of the KLF-4 promoter and downregulation of KLF-4 and NOS3 in the endothelium of OS regions in swine. At the same time, Jo et al. [125] and Chien et al. [126] demonstrated that DNMT1 is modulated by OS, resulting in induction of EC dysfunction. Moreover, DNMT-1 is upregulated by OS to regulate EC inflammation in vitro. A partial carotid ligation mouse model was used to generate OS in vivo, confirming that DNMT-1 is overexpressed in ECs in response to OS.

Using both reduced representation bisulfite sequencing and microarray analysis, researchers found that hypermethylation occurred in the promoters of 11 mechanosensitive genes in ECs in response to OS. Among these 11 mechanosensitive genes, HOXA5 is an important transcription factor that modulates inflammation. Thus, OS may mediate the methylation of promoters of mechanosensitive genes, including the transcription factor HOX5, to regulate OS-mediated pro-inflammatory responses [125]. Chien and colleagues [126] showed that OS induces not only the expression and nuclear accumulation of DNMT-1, but also the hypermethylation of DNA. Inhibition of DNMT-1 by 5-aza-2'-deoxycytidine (5Aza, also known as decitabine) suppresses OS-induced DNA hypermethylation. In concert with these findings, in vivo results showed that DNMT1 expression and DNA methylation are increased in OS regions of partially ligated rat carotid arteries [126]. Mechanistically, Zhang et al. [127] showed that OS-dependent induction of DNMT1 is modulated by integrin/Shc/focal adhesion kinase/extracellular signal-regulated kinase/mTOR/p70S6K signaling pathways. Moreover, OS-induced DNMT1 results in upregulation of cyclinA and connective tissue growth factor, which modulate EC proliferation and inflammation, respectively. These studies suggest that DNMT1 is involved in OS-induced EC dysfunction in vitro, including aberrant EC proliferation, inflammation, and $\mathrm{NO}$ repression.

DNMTs are also related to vascular disorders, including atherosclerosis. Jo and colleagues [125] showed that DNMT1 is correlated with atherosclerosis. In a partial carotid ligation mouse model, treatment of $\mathrm{ApoE}^{-1-}$ mice with $5 \mathrm{Aza}$ inhibits the formation of atherosclerosis. Zhang et al. [127] further used an $\mathrm{ApoE}^{-/-}$mouse model to demonstrate that DNMT1 overexpression and DNA hypermethylation occur in the endothelium of atherosclerotic lesions. Silencing of DNMT-1 by adenovirusmediated DNMT shRNA inhibits the expressions of EC dysfunction-related proteins, including proliferating cell nuclear antigen, VCAM-1, and ICAM-1, and blocks the development of atherosclerosis.

\section{Summary and conclusion}

EC dysfunction, e.g., turnover enrichment, inflammation, and oxidation, is an important step for initiation of vascular disorder such as atherosclerosis. Vascular ECs are subjected to blood flow to activate mechanotransduction, which regulates EC function and dysfunction. Proatherogenic OS can modulate various signaling pathways to induce EC dysfunction and promote atherosclerosis. In contrast, atheroprotective PS can modulate various signaling pathways to inhibit EC dysfunction and protect against atherosclerosis.

Epigenetics has been emerged as a new field in vascular biology. In recent studies, epigenetic factors, including HDACs, non-coding RNAs, and DNMTs, have been shown to be involved in hemodynamic force-modulated EC function and dysfunction. In this review, we summarized current studies on the roles of these epigenetic factors in hemodynamics-modulated EC function and dysfunction, and hence atherosclerosis. Moreover, we discussed the 
Table 3 Roles of DNMTs in hemodynamics-modulated endothelial function and dysfunction

\begin{tabular}{|c|c|c|c|c|c|}
\hline & DNMTs & Target & Affected molecule & EC dysfunction/function & Ref \\
\hline \multirow[t]{9}{*}{ Pro-atherogenic OS } & DNMT3a $\uparrow$ & 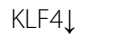 & 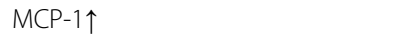 & Inflammation & 124 \\
\hline & & & Thrombomodulin $\downarrow$ & Inflammation & 124 \\
\hline & & & NOS3 $\downarrow$ & NO repression & 124 \\
\hline & DNMT1个 & HoxA5 $\downarrow$ & Monocyte adhesion-related genes $\uparrow$ & Inflammation & 125 \\
\hline & & $?$ & VCAM-1个 & Inflammation & 127 \\
\hline & & $?$ & ICAM-1个 & Inflammation & 127 \\
\hline & & $\mathrm{CTGF} \uparrow$ & Monocyte adhesion-related genes $\uparrow$ & Inflammation & 127 \\
\hline & & $?$ & PCNA $\uparrow$ & Proliferation & 127 \\
\hline & & CyclinA $A$ & $?$ & Proliferation & 127 \\
\hline
\end{tabular}

detailed mechanisms by which mechanotransduction regulates epigenetic factors to affect EC function and dysfunction in response to various hemodynamic forces, i.e., proatherogenic OS and atheroprotective PS. Furthermore, we elucidated the relationship between epigenetic factors and vascular disorders, i.e., atherosclerosis. We discussed the mechanisms by which class I and II HDACs alter the expression of proliferative, pro-inflammatory, and oxidative signaling molecules to regulate EC function and dysfunction in response to differential hemodynamic forces. Proatherogenic OS induces the expression and nuclear accumulation of class I and II HDACs to induce EC dysfunction, whereas atheroprotective PS induces phosphorylationdependent nuclear export of class II HDACs to inhibit EC dysfunction. In addition, class III HDACs, e.g., Sirt1, are induced by atheroprotective PS, resulting in acceleration of NO production.

We also discussed the novel mechanisms by which hemodynamic forces transcriptionally regulate miRs. Atheroprotective PS induces the expression, nuclear accumulation, and association of the hormone receptors RAR $\alpha$ and RXR $\alpha$ to activate miR-10a-directed anti-inflammatory signaling. In contrast, pro-athergenic OS induces the association of HDAC-3/-5/-7 and RAR $\alpha$ to form a repression complex and inhibit miR-10a-directed anti-inflammatory signaling. In addition, flow-modulated KLF-2 regulates several miRs, including miR-126 and $m i R-23 b$. We summarized current studies showing how pro-atherogenic OS modulates miRs to activate proliferative, pro-inflammatory, and oxidative signaling and induce EC dysfunction, whereas atheroprotective PS modulates an array of miRs to drive antiproliferative, anti-inflammatory, anti-oxidative, and NO-related signaling and prevent EC dysfunction. Moreover, we provided new information that PS can modulate lncRNAs to regulate EC function, including cell turnover, migration, angiogenesis, and cell-cell interaction. Finally, proatherogenic OS has been shown to induce the expression of DNMT1 and DNMT3a and subsequently modulates EC dysfunction, i.e., proliferation, inflammation, and NO repression. All of these studies indicate that epigenetic factors, i.e., HDACs, miRs, lncRNAs, and DNMTs, are involved in hemodynamics-directed EC function and dysfunction and hence atherosclerosis. Understanding the relationship between epigenetic factors and EC function and dysfunction under pro-atherogenic or atheroprotective flow conditions will help to elucidate the pathogenic mechanisms of vascular disorders, such as atherosclerosis. In addition, the information provided in this review will help us to identify potential targets, which will facilitate the development of new strategies for the treatment of atherosclerosis.

\section{Abbreviations \\ 5Aza: 5-aza-2'-deoxycytidine; 5mC: 5-methylcytosine; $\mathrm{ApoE}^{-/-}$: Apolipoprotein E-deficient; CircRNAs: Circular RNAs; Cx: Connexins; DNMT: DNA methyltransferase; EC: Endothelial cell; eNOS: Endothelial nitric oxide synthase; Gpx: Glutathione peroxidase; HDAC: Histone deacetylase; ICAM-1: Intercellular adhesion molecule-1; KLF-2: Krüppel like factor 2; LDL: Low-density lipoprotein; IncRNAs: Long noncoding RNAs; MBD2: methyl-CpG-binding domain protein 2; MCP- \\ 1: Monocyte chemoattractant protein-1; MEF2: Myocyte enhancer factor 2; MiR: microRNA; mTOR: Mammalian target of rapamycin; NAD+: Nicotinamide adenine dinucleotide; NF: Nuclear factor; NO: Nitric oxide; NQO1: NADPH quinine oxidoreductase 1; Nrf2: NF-E2-related factor 2; OS: Oscillatory shear stress; PPAR: Peroxisome proliferator-activated receptor; Pre-miR: Precursor miR; Pri-miR: Primary miR; PS: Pulsatile shear stress; RAR: Retinoic acid receptor; ROS: Reactive oxygen species; RXR: Retinoid X receptor; Sirt: Sirtuin; SOD: Superoxide dismutase; TM: Thrombomodulin; UTR: Untranslated region; VCAM-1: Vascular cell adhesion molecule - 1; VE: Vascular endothelial; VEGF-R2: Vascular endotheilial growth factor receptor 2}

Acknowledgements

Not applicable.

Authors' contributions

DYL and JJC wrote the manuscript, discussed the content, and approved the final version of manuscript.

\section{Funding}

This work was supported by the Ministry of Science and Technology, Taiwan (Grants MOST-108-2633-B-009-001/107-2321-B-400-001, to J.-J.C., MOST-1082320-B-157-001/ 107-2320-B-157-001, to D.-Y.-L.), National Health Research Institutes, and Central Government S \& T grants (108-1901-01-19-08/1081901-01-19-07/107-1901-01-19-03/ MOST106-3114-Y-043-021/108-0324-0119-07/107-0324-01-19-03/106-0324-01-10-07/105-0324-01-10-03, to J.-J.C.).

Availability of data and materials Not applicable. 


\section{Ethics approval and consent to participate}

Not applicable.

\section{Consent for publication}

Not applicable.

\section{Competing interests}

The authors declare that they have no competing interests.

\section{Author details}

'Department of Biological Science and Technology, China University of Science and Technology, Taipei 115, Taiwan. ${ }^{2}$ Institute of Cellular and System Medicine, National Health Research Institutes, Miaoli 350, Taiwan. ${ }^{3}$ Institute of Biomedical Engineering, National Tsing Hua University, Hsinchu 300, Taiwan. ${ }^{4}$ Collage of Pharmacy, Taipei Medical University, Taipei 110, Taiwan. ${ }^{5}$ Institute of Biomedical Engineering, National Cheng Kung University, Tainan 701, Taiwan. ${ }^{6}$ Institute of Polymer Science and Engineering, National Taiwan University, Taipei 106, Taiwan.

Received: 8 April 2019 Accepted: 29 July 2019

Published online: 07 August 2019

\section{References}

1. Chiu JJ, Chien S. Effects of disturbed flow on vascular endothelium: pathophysiological basis and clinical perspectives. Physiol Rev. 2011; 91(1):327-87.

2. Davies PF. Flow-mediated endothelial mechanotransduction. Physiol Rev. 1995;75(3):519-60.

3. Zhou J, Li YS, Chien S. Shear stress-initiated signaling and its regulation of endothelial function. Arterioscler Thromb Vasc Biol. 2014;34(10):2191-8.

4. Ross R, Glomset J. The pathogenesis of atherosclerosis. N Engl J Med. 1976; 295(7):369-77.

5. Bonetti PO, Lerman LO, Lerman A. Endothelial dysfunction: a marker of atherosclerotic risk. Arterioscler Thromb Vasc Biol. 2003;23(2):168-75.

6. Weber $\mathrm{C}$, Noels $\mathrm{H}$. Atherosclerosis: current pathogenesis and therapeutic options. Nat Med. 2011;17(11):1410-22.

7. Mundi S, Massaro M, Scoditti E, Carluccio MA, van Hinsbergh WWM, IruelaArispe ML, De Caterina R. Endothelial permeability, LDL deposition, and cardiovascular risk factors-a review. Cardiovasc Res. 2018;114(1):35-52.

8. Weinbaum S, Tzeghai G, Ganatos P, Pfeffer R, Chien S. Effect of cell turnover and leaky junctions on arterial macromolecular transport. Am J Phys. 1985; 248:H945-60.

9. Li YS, Haga JH, Chien S. Molecular basis of the effects of shear stress on vascular endothelial cells. J Biomech. 2005;38(10):1949-71.

10. Zhou J, Lee PL, Tsai CS, Lee Cl, Yang TL, Chuang HS, Lin WW, Lin TE, Lim SH, Wei SY, Chen YL, Chien S, Chiu JJ. Force-specific activation of Smad1/5 regulates vascular endothelial cell cycle progression in response to disturbed flow. Proc Natl Acad Sci U S A. 2012:109(20):7770-5.

11. Akimoto S, Mitsumata M, Sasaguri T, Yoshida Y. Laminar shear stress inhibits vascular endothelial cell proliferation by inducing cyclin-dependent kinase inhibitor p21 Sdi1/Cip1/Waf1. Circ Res. 2000;86(2):185-90.

12. Chien S. Molecular and mechanical bases of focal lipid accumulation in arterial wall. Prog Biophys Mol Biol. 2003:83(2):131-51.

13. Guo D, Chien S, Shyy JY. Regulation of endothelial cell cycle by laminar versus oscillatory flow: distinct modes of interactions of AMP-activated protein kinase and Akt pathways. Circ Res. 2007;100(4):564-71.

14. Miao H, Hu YL, Shiu YT, Yuan S, Zhao Y, Kaunas R, Wang Y, Jin G, Usami S, Chien S. Effects of flow patterns on the localization and expression of VEcadherin at vascular endothelial cell junctions: in vivo and in vitro investigations. J Vasc Res. 2005;42(1):77-89.

15. DePaola N, Davies PF, Pritchard WF, Florez L, Harbeck N, Polacek DC. Spatial and temporal regulation of gap junction connexin43 in vascular endothelial cells exposed to controlled disturbed flows in vitro. Proc Natl Acad Sci U S A. 1999;96(6):3154-9.

16. Libby P. Inflammation in atherosclerosis. Nature. 2002;420(6917):868-74.

17. Mohan S, Mohan N, Sprague EA. Differential activation of NF-kappa B in human aortic endothelial cells conditioned to specific flow environments. Am J Phys. 1997;273(2 Pt 1):C572-8.

18. Dardik A, Chen L, Frattini J, Asada H, Aziz F, Kudo FA, Sumpio BE. Differential effects of orbital and laminar shear stress on endothelial cells. J Vasc Surg. 2005;41(5):869-80
19. Chappell DC, Varner SE, Nerem RM, Medford RM, Alexander RW. Oscillatory shear stress stimulates adhesion molecule expression in cultured human endothelium. Circ Res. 1998;82(5):532-9.

20. Sorescu GP, Sykes M, Weiss D, Platt MO, Saha A, Hwang J, Boyd N, Boo YC, Vega JD, Taylor WR, Jo H. Bone morphogenic protein 4 produced in endothelial cells by oscillatory shear stress stimulates an inflammatory response. J Biol Chem. 2003;278(33):31128-35.

21. Lubrano V, Balzan S. Enzymatic antioxidant system in vascular inflammation and coronary artery disease. World J Exp Med. 2015;5(4):218-24.

22. Hosoya T, Maruyama A, Kang MI, Kawatani Y, Shibata T, Uchida K, Warabi E, Noguchi N, Itoh K, Yamamoto M. Differential responses of the Nrf2-Keap1 system to laminar and oscillatory shear stresses in endothelial cells. J Biol Chem. 2005:280(29):27244-50.

23. Topper JN, Cai J, Falb D, Gimbrone MA Jr. Identification of vascular endothelial genes differentially responsive to fluid mechanical stimuli: cyclooxygenase-2, manganese superoxide dismutase, and endothelial cell nitric oxide synthase are selectively up-regulated by steady laminar shear stress. Proc Natl Acad Sci U S A. 1996;93(19):10417-22.

24. Frangos JA, Eskin SG, Mclntire LV, Ives CL. Flow effects on prostacyclin production by cultured human endothelial cells. Science. 1985;227(4693): 1477-9.

25. Wang J, Pan S, Berk BC. Glutaredoxin mediates Akt and eNOS activation by flow in a glutathione reductase-dependent manner. Arterioscler Thromb Vasc Biol. 2007;27(6):1283-8.

26. Goldberg $A D$, Allis $C D$, Bernstein E. Epigenetics: a landscape takes shape. Cell. 2007;128(4):635-8

27. Zhou B, Margariti A, Zeng L, Xu Q. Role of histone deacetylases in vascular cell homeostasis and arteriosclerosis. Cardiovasc Res. 2011:90(3):413-20.

28. Condorelli G, Latronico MV, Cavarretta E. microRNAs in cardiovascular diseases: current knowledge and the road ahead. J Am Coll Cardiol. 2014; 63(21):2177-87.

29. Kumar S, Kim CW, Simmons RD, Jo H. Role of flow-sensitive microRNAs in endothelial dysfunction and atherosclerosis: mechanosensitive athero-miRs. Arterioscler Thromb Vasc Biol. 2014;34(10):2206-16.

30. Marin T, Gongol B, Chen Z, Woo B, Subramaniam S, Chien S, Shyy JY. Mechanosensitive microRNAs-role in endothelial responses to shear stress and redox state. Free Radic Biol Med. 2013;64:61-8.

31. Dunn J, Thabet $\mathrm{S}$, Jo H. Flow-dependent epigenetic DNA methylation in endothelial gene expression and atherosclerosis. Arterioscler Thromb Vasc Biol. 2015:35(7):1562-9.

32. Wade PA. Transcriptional control at regulatory checkpoints by histone deacetylases : molecular connections between cancer and chromatin. Hum Mol Genet. 2001:10(7):693-8.

33. de Ruijter AJ, van Gennip AH, Caron HN, Kemp S, van Kuilenburg AB. Histone deacetylases (HDACs): characterization of the classical HDAC family. Biochem J. 2003;370(3):737-49.

34. Delcuve GP, Khan DH, Davie JR. Roles of histone deacetylases in epigenetic regulation: emerging paradigms from studies with inhibitors. Clin Epigenetics. 2012;4(1):5.

35. Marks PA. Histone deacetylase inhibitors: a chemical genetics approach to understanding cellular functions. Biochim Biophys Acta. 2010;1799(10-12):717-25.

36. Verdin E, Dequiedt F, Kasler HG. Class II histone deacetylases: versatile regulators. Trends Genet. 2003;19(5):286-93.

37. Parra M, Verdin E. Regulatory signal transduction pathways for class Ila histone deacetylases. Curr Opin Pharmacol. 2010;10(4):454-60.

38. Li G, Jiang H, Chang M, Xie H, Hu L. HDAC6 a-tubulin deacetylase: a potential therapeutic target in neurodegenerative diseases. J Neurol Sci. 2011;304(1-2):1-8

39. Haigis MC, Guarente LP. Mammalian sirtuins-emerging roles in physiology, aging, and calorie restriction. Genes Dev. 2006;20(21):2913-21.

40. Villagra A, Sotomayor EM, Seto E. Histone deacetylases and the immunological network: implications in cancer and inflammation. Oncogene. 2010;29(2):157-73.

41. Montgomery RL, Davis CA, Potthoff MJ, Haberland M, Fielitz J, Qi X, Hill JA, Richardson JA, Olson EN. Histone deacetylases 1 and 2 redundantly regulate cardiac morphogenesis, growth, and contractility. Genes Dev. 2007;21(14): 1790-802.

42. Zampetaki A, Zeng L, Margariti A, Xiao Q, Li H, Zhang Z, Pepe AE, Wang G, Habi O, de Falco E, Cockerill G, Mason JC, Hu Y, Xu Q. Histone deacetylase 3 is critical in endothelial survival and atherosclerosis development in response to disturbed flow. Circulation. 2010;121(1):132-42. 
43. Chang S, McKinsey TA, Zhang CL, Richardson JA, Hill JA, Olson EN. Histone deacetylases 5 and 9 govern responsiveness of the heart to a subset of stress signals and play redundant roles in heart development. Mol Cell Biol. 2004;24(19):8467-76.

44. Chang S, Young BD, Li S, Qi X, Richardson JA, Olson EN. Histone deacetylase 7 maintains vascular integrity by repressing matrix metalloproteinase 10 . Cell. 2006;126(2):321-34.

45. Stein S, Matter CM. Protective roles of SIRT1 in atherosclerosis. Cell Cycle. 2011;10(4):640-7.

46. Zeng L, Zhang Y, Chien S, Liu X, Shyy JY. The role of p53 deacetylation in p21Waf1 regulation by laminar flow. J Biol Chem. 2003;278(27):24594-9.

47. Zeng L, Xiao Q, Margariti A, Zhang Z, Zampetaki A, Patel S, Capogrossi MC, $\mathrm{Hu} Y, X \cup \mathrm{Q} . \mathrm{HDAC3}$ is crucial in shear- and VEGF-induced stem cell differentiation toward endothelial cells. J Cell Biol. 2006;174(7):1059-69.

48. Lee DY, Lee Cl, Lin TE, Lim SH, Zhou J, Tseng YC, Chien S, Chiu JJ. Role of histone deacetylases in transcription factor regulation and cell cycle modulation in endothelial cells in response to disturbed flow. Proc Natl Acad Sci U S A. 2012;109(6):1967-72.

49. Boon RA, Horrevoets AJ. Key transcriptional regulators of the vasoprotective effects of shear stress. Hamostaseologie. 2009;29(1):39-43.

50. Wang $\mathrm{W}, \mathrm{Ha} \mathrm{CH}$, Jhun BS, Wong $\mathrm{C}$, Jain MK, Jin ZG. Fluid shear stress stimulates phosphorylation-dependent nuclear export of HDAC5 and mediates expression of KLF2 and eNOS. Blood. 2010;115(14):2971-9.

51. Wang $Y H$, Yan ZQ, Qi YX, Cheng BB, Wang XD, Zhao D, Shen BR, Jiang ZL. Normal shear stress and vascular smooth muscle cells modulate migration of endothelial cells through histone deacetylase 6 activation and tubulin acetylation. Ann Biomed Eng. 2010;38(3):729-37.

52. Chen Z, Peng IC, Cui X, Li YS, Chien S, Shyy JY. Shear stress, SIRT1, and vascular homeostasis. Proc Natl Acad Sci U S A. 2010;107(22):10268-73.

53. Eom GH, Kook H. Posttranslational modifications of histone deacetylases: implications for cardiovascular diseases. Pharmacol Ther. 2014;143(2):168-80.

54. Zheng XX, Zhou T, Wang XA, Tong XH, Ding JW. Histone deacetylases and atherosclerosis. Atherosclerosis. 2015;240(2):355-66.

55. Kee HJ, Kwon JS, Shin S, Ahn Y, Jeong MH, Kook H. Trichostatin a prevents neointimal hyperplasia via activation of Krüppel like factor 4. Vasc Pharmacol. 2011;55(5-6):127-34.

56. Findeisen HM, Gizard F, Zhao Y, Qing H, Heywood EB, Jones KL, Cohn D, Bruemmer D. Epigenetic regulation of vascular smooth muscle cell proliferation and neointima formation by histone deacetylase inhibition. Arterioscler Thromb Vasc Biol. 2011;31(4):851-60.

57. Usui T, Morita T, Okada M, Yamawaki H. Histone deacetylase 4 controls neointimal hyperplasia via stimulating proliferation and migration of vascular smooth muscle cells. Hypertension. 2014;63(2):397-403.

58. Zhang QJ, Wang Z, Chen HZ, Zhou S, Zheng W, Liu G, Wei YS, Cai H, Liu DP, Liang CC. Endothelium-specific overexpression of class III deacetylase SIRT1 decreases atherosclerosis in apolipoprotein E-deficient mice. Cardiovasc Res. 2008;80(2):191-9.

59. Xu S, Yin M, Koroleva M, Mastrangelo MA, Zhang W, Bai P, Little PJ, Jin ZG. SIRT6 protects against endothelial dysfunction and atherosclerosis in mice. Aging (Albany NY). 2016;8(5):1064-82.

60. Lee DY, Lin TE, Lee Cl, Zhou J, Huang YH, Lee PL, Shih YT, Chien S, Chiu JJ. MicroRNA-10a is crucial for endothelial response to different flow patterns via interaction of retinoid acid receptors and histone deacetylases. Proc Natl Acad Sci U S A. 2017;114(8):2072-7.

61. Nicoli S, Standley C, Walker P, Hurlstone A, Fogarty KE, Lawson ND. MicroRNAmediated integration of haemodynamics and Vegf signaling during angiogenesis. Nature. 2010;464(7292):1196-200.

62. Bartel DP. MicroRNAs: genomics, biogenesis, mechanism, and function. Cell. 2004;116(2):281-97.

63. Giraldez AJ, Cinalli RM, Glasner ME, Enright AJ, Thomson JM, Baskerville S, Hammond SM, Bartel DP, Schier AF. MicroRNAs regulate brain morphogenesis in zebrafish. Science. 2005;308(5723):833-8.

64. Yang WJ, Yang DD, Na S, Sandusky GE, Zhang Q, Zhao G. Dicer is required for embryonic angiogenesis during mouse development. J Biol Chem. 2005; 280(10):9330-5.

65. Chen JF, Murchison EP, Tang R, Callis TE, Tatsuguchi M, Deng Z, Rojas M, Hammond SM, Schneider MD, Selzman CH, Meissner G, Patterson C, Hannon GJ, Wang DZ. Targeted deletion of dicer in the heart leads to dilated cardiomyopathy and heart failure. Proc Natl Acad Sci U S A. 2008;105(6):2111-6.

66. da Costa Martins PA, Bourajiaj M, Gladka M, Kortland M, van Oort RJ, Pinto YM, Molkentin JD, De Windt LJ. Conditional dicer gene deletion in the postnatal myocardium provokes spontaneous cardiac remodeling. Circulation. 2008;118(15):1567-76

67. Wang H, Zhu HQ, Wang F, Zhou Q, Gui SY, Wang Y. MicroRNA-1 prevents high-fat diet-induced endothelial permeability in apoE knock-out mice. Mol Cell Biochem. 2013;378(1-2):153-9.

68. Liu N, Bezprozvannaya S, Williams AH, Qi X, Richardson JA, Bassel-Duby R, Olson EN. microRNA-133a regulates cardiomyocyte proliferation and suppresses smooth muscle gene expression in the heart. Genes Dev. 2008;22(23):3242-54

69. Fish JE, Santoro MM, Morton SU, Yu S, Yeh RF, Wythe JD, Ivey KN, Bruneau BG, Stainier DY, Srivastava D. miR-126 regulates angiogenic signaling and vascular integrity. Dev Cell. 2008;15(2):272-84

70. Wang S, Aurora AB, Johnson BA, Qi X, McAnally J, Hill JA, Richardson JA, Bassel-Duby R, Olson EN. The endothelial-specific microRNA miR-126 governs vascular integrity and angiogenesis. Dev Cell. 2008;15(2):261-71.

71. Oin X, Wang X, Wang Y, Tang Z, Cui O, Xi J, Li YS, Chien S, Wang N. Microrna-19a mediates the suppressive effect of laminar flow on cyclin d1 expression in human umbilical vein endothelial cells. Proc Natl Acad Sci U S A. 2010;107(7):3240-4.

72. Wang KC, Garmire LX, Young A, Nguyen P, Trinh A, Subramaniam S, Wang $\mathrm{N}$, Shyy JY, Li YS, Chien S. Role of microrna-23b in flow-regulation of rb phosphorylation and endothelial cell growth. Proc Natl Acad Sci U S A. 2010;107(7):3234-9.

73. Ni CW, Qiu H, Jo H. Microrna-663 upregulated by oscillatory shear stress plays a role in inflammatory response of endothelial cells. Am J Physiol Heart Circ Physiol. 2011;300(5):H1762-9.

74. Fang Y, Shi C, Manduchi E, Civelek M, Davies PF. Microrna-10a regulation of proinflammatory phenotype in athero-susceptible endothelium in vivo and in vitro. Proc Natl Acad Sci U S A. 2010;107(30):13450-5.

75. Son DJ, Kumar S, Takabe W, Kim CW, Ni CW, Alberts-Grill N, Jang $H_{\text {, }}$ Kim S, Kim W, Won Kang S, Baker AH, Woong Seo J, Ferrara KW, Jo H. The atypical mechanosensitive microrna-712 derived from pre-ribosomal rna induces endothelial inflammation and atherosclerosis. Nat Commun. 2013:4:3000.

76. Lee DY, Yang TL, Huang YH, Lee Cl, Chen LJ, Shih YT, Wei SY, Wang WL, Wu CC, Chiu JJ. Induction of microRNA-10a using retinoic acid receptor-a and retinoid $\mathrm{x}$ receptor-a agonists inhibits atherosclerotic lesion formation. Atherosclerosis. 2018:271:36-44.

77. Wang N, Miao H, Li YS, Zhang P, Haga JH, Hu Y, Young A, Yuan S, Nguyen P. Wu CC, Chien S. Shear stress regulation of Krüppel-like factor 2 expression is flow pattern-specific. Biochem Biophys Res Commun. 2006;341(4):1244-51.

78. Wang KC, Nguyen $P$, Weiss A, Yeh YT, Chien HS, Lee A, Teng D, Subramaniam S, Li YS, Chien S. MicroRNA-23b regulates cyclin-dependent kinase-activating kinase complex through cyclin $\mathrm{H}$ repression to modulate endothelial transcription and growth under flow. Arterioscler Thromb Vasc Biol. 2014;34(7):1437-45.

79. Chen K, Fan W, Wang X, Ke X, Wu G, Hu C. MicroRNA-101 mediates the suppressive effect of laminar shear stress on mTOR expression in vascular endothelial cells. Biochem Biophys Res Commun. 2012:427(1):138-42.

80. Weber M, Kim S, Patterson N, Rooney K, Searles CD. MiRNA-155 targets myosin light chain kinase and modulates actin cytoskeleton organization in endothelial cells. Am J Physiol Heart Circ Physiol. 2014;306(8):H1192-203.

81. Schober A, Nazari-Jahantigh M, Wei Y, Bidzhekov K, Gremse F, Grommes J, Megens RT, Heyll K, Noels H, Hristov M, Wang S, Kiessling F, Olson EN, Weber C. Microrna-126-5p promotes endothelial proliferation and limits atherosclerosis by suppressing dlk1. Nat Med. 2014;20(4):368-76.

82. Wu W, Xiao H, Laguna-Fernandez A, Villarreal G Jr, Wang KC, Geary GG, Zhang Y, Wang WC, Huang HD, Zhou J, Li YS, Chien S, Garcia-Cardena G, Shyy JY. Flow-dependent regulation of kruppel-like factor 2 is mediated by microRNA-92a. Circulation. 2011;124(5):633-41.

83. Zhou J, Wang KC, Wu W, Subramaniam S, Shyy JY, Chiu JJ, Li JY, Chien S. Microrna-21 targets peroxisome proliferators-activated receptor-alpha in an autoregulatory loop to modulate flow-induced endothelial inflammation. Proc Natl Acad Sci U S A. 2011;108(25):10355-60.

84. Fan W, Fang R, Wu X, Liu J, Feng M, Dai G, Chen G, Wu G. Shear-sensitive microRNA-34a modulates flow-dependent regulation of endothelial inflammation. J Cell Sci. 2015;128(1):70-80.

85. Chen LJ, Chuang L, Huang YH, Zhou J, Lim SH, Lee Cl, Lin WW, Lin TE, Wang WL, Chen L, Chien S, Chiu JJ. MicroRNA mediation of endothelial inflammatory response to smooth muscle cells and its inhibition by atheroprotective shear stress. Circ Res. 2015;116(7):1157-69.

86. Deanfield JE, Halcox JP, Rabelink TJ. Endothelial function and dysfunction: testing and clinical relevance. Circulation. 2007;115(10):1285-95 Review. 
87. Weber M, Baker MB, Moore JP, Searles CD. MiR-21 is induced in endothelial cells by shear stress and modulates apoptosis and eNOS activity. Biochem Biophys Res Commun. 2010;393(4):643-8.

88. Loyer X, Potteaux S, Vion AC, Guérin CL, Boulkroun S, Rautou PE, Ramkhelawon B, Esposito B, Dalloz M, Paul JL, Julia P, Maccario J, Boulanger CM, Mallat Z, Tedgui A. Inhibition of microRNA-92a prevents endothelial dysfunction and atherosclerosis in mice. Circ Res. 2014;114(3):434-43.

89. Kumar S, Williams D, Sur S, Wang JY, Jo H. Role of flow-sensitive microRNAs and long noncoding RNAs in vascular dysfunction and atherosclerosis. Vasc Pharmacol. 2019;114:76-92

90. Simion V, Haemmig S, Feinberg MW. LncRNAs in vascular biology and disease. Vasc Pharmacol. 2019;114:145-56.

91. Bei $Y$, Yang T, Wang L, Holvoet P, Das S, Sluijter JPG, Monteiro MC, Liu Y, Zhou Q, Xiao J. Circular RNAs as potential theranostics in the cardiovascular system. Mol Ther Nucleic Acids. 2018;13:407-18.

92. Zhou MY, Yang JM, Xiong XD. The emerging landscape of circular RNA in cardiovascular diseases. J Mol Cell Cardiol. 2018;122:134-9.

93. Man HSJ, Sukumar AN, Lam GC, Turgeon PJ, Yan MS, Ku KH, Dubinsky MK, Ho JJD, Wang JJ, Das S, Mitchell N, Oettgen P, Sefton MV, Marsden PA. Angiogenic patterning by STEEL, an endothelial-enriched long noncoding RNA. Proc Natl Acad Sci U S A. 2018;115(10):2401-6.

94. Leisegang MS, Fork C, Josipovic I, Richter FM, Preussner J, Hu J, Miller MJ, Epah J, Hofmann P, Günther S, Moll F, Valasarajan C, Heidler J, Ponomareva Y, Freiman TM, Maegdefessel L, Plate KH, Mittelbronn M, Uchida S, Künne C, Stellos K, Schermuly RT, Weissmann N, Devraj K, Wittig I, Boon RA, Dimmeler S, Pullamsetti SS, Looso M, Miller FJ Jr, Brandes RP. Long noncoding RNA MANTIS facilitates endothelial angiogenic function. Circulation. 2017;136(1): 65-79.

95. Stanicek L, Lozano-Vidal N, Kwon HB, Stainier D, Wittig I, Dimmeler S, Boon RA. Abstract 19863: the shear stress-induced human long non-coding RNA lassie regulates endothelial cell-cell junctions. Circulation. 2017;136(Suppl 1): A19863.

96. Josipovic I, Pflüger B, Fork C, Vasconez AE, Oo JA, Hitzel J, Seredinski S, Gamen E, Heringdorf DMZ, Chen W, Looso M, Pullamsetti SS, Brandes RP, Leisegang MS. Long noncoding RNA LISPR1 is required for S1P signaling and endothelial cell function. J Mol Cell Cardiol. 2018;116:57-68.

97. Pan JX. LncRNA H19 promotes atherosclerosis by regulating MAPK and NFKB signaling pathway. Eur Rev Med Pharmacol Sci. 2017;21(2):322-8.

98. Bao MH, Luo HQ, Chen LH, Tang L, Ma KF, Xiang J, Dong LP, Zeng J, Li GY, Li JM. Impact of high fat diet on long non-coding RNAs and messenger RNAs expression in the aortas of ApoE(-/-) mice. Sci Rep. 2016;6:34161.

99. Zhang Z, Gao W, Long QQ, Zhang J, Li YF, Liu DC, Yan JJ, Yang ZJ, Wang LS. Increased plasma levels of IncRNA H19 and LIPCAR are associated with increased risk of coronary artery disease in a Chinese population. Sci Rep. 2017;7(1):7491.

100. Boulberdaa M, Scott E, Ballantyne M, Garcia R, Descamps B, Angelini GD, Brittan M, Hunter A, McBride M, McClure J, Miano JM, Emanueli C, Mills NL, Mountford JC, Baker AH. A role for the long noncoding RNA SENCR in commitment and function of endothelial cells. Mol Ther. 2016;24(5):978-90.

101. Wu Z, He Y, Li D, Fang X, Shang T, Zhang H, Zheng X. Long noncoding RNA MEG3 suppressed endothelial cell proliferation and migration through regulating miR-21. Am J Transl Res. 2017;9(7):3326-35.

102. Shan $K$, Jiang $Q$, Wang $X Q$, Wang YN, Yang H, Yao MD, Liu C, Li XM, Yao J, Liu B, Zhang YY, Yong J, Yan B. Role of long non-coding RNA-RNCR3 in atherosclerosis-related vascular dysfunction. Cell Death Dis. 2016;7(6):e2248.

103. Han DK, Khaing ZZ, Pollock RA, Haudenschild CC, Liau G. H19, a marker of developmental transition, is reexpressed in human atherosclerotic plaques and is regulated by the insulin family of growth factors in cultured rabbit smooth muscle cells. J Clin Invest. 1996;97:1276-85.

104. Dang RY, Liu FL, Li Y. Circular RNA hsa_circ_0010729 regulates vascular endothelial cell proliferation and apoptosis by targeting the miR-186/HIF-1a axis. Biochem Biophys Res Commun. 2017:490:104-10.

105. Liu C, Yao MD, Li CP, Shan K, Yang H, Wang JJ, Liu B, Li XM, Yao J, Jiang Q, Yan B. Silencing of circular RNA-ZNF609 ameliorates vascular endothelial dysfunction. Theranostics. 2017;7:2863-77.

106. Holdt LM, Stahringer A, Sass K, Pichler G, Kulak NA, Wilfert W, Kohlmaier A, Herbst A, Northoff BH, Nicolaou A, Gäbel G, Beutner F, Scholz M, Thiery J, Musunuru K, Krohn K, Mann M, Teupser D. Circular non-coding RNA ANRIL modulates ribosomal RNA maturation and atherosclerosis in humans. Nat Commun. 2016;7:12429.

107. Song CL, Wang JP, Xue X, Liu N, Zhang XH, Zhao Z, Liu JG, Zhang CP, Piao $Z \mathrm{H}$, Liu Y, Yang YB. Effect of circular ANRIL on the inflammatory response of vascular endothelial cells in a rat model of coronary atherosclerosis. Cel Physiol Biochem. 2017;42(3):1202-12.

108. Zhao Z, Li X, Gao C, Jian D, Hao P, Rao L, Li M. Peripheral blood circular RNA hsa_circ_0124644 can be used as a diagnostic biomarker of coronary artery disease. Sci Rep. 2017;7:39918.

109. Pan RY, Liu P, Zhou HT, Sun WX, Song J, Shu J, Cui GJ, Yang ZJ, Jia EZ Circular RNAs promote TRPM3 expression by inhibiting hsa-miR-130a-3p in coronary artery disease patients. Oncotarget. 2017;8:60280-90.

110. Moore LD, Le T, Fan G. DNA methylation and its basic function. Neuropsychopharmacology. 2013;38(1):23-38.

111. Clouaire T, de Las Heras JI, Merusi C, Stancheva I. Recruitment of mbd1 to target genes requires sequence-specific interaction of the mbd domain with methylated DNA. Nucleic Acids Res. 2010;38(14):4620-34.

112. Portela A, Esteller M. Epigenetic modifications and human disease. Nat Biotechnol. 2010;28(10):1057-68.

113. Shirodkar AV, Marsden PA. Epigenetics in cardiovascular disease. Curr Opin Cardiol. 2011;26(3):209-15.

114. Dunn J, Simmons R, Thabet $S$, Jo H. The role of epigenetics in the endothelial cell shear stress response and atherosclerosis. Int J Biochem Cell Biol. 2015;67: 167-76.

115. Jiang YZ, Manduchi E, Jiménez JM, Davies PF. Endothelial epigenetics in biomechanical stress: disturbed flow-mediated epigenomic plasticity in vivo and in vitro. Arterioscler Thromb Vasc Biol. 2015;35(6):1317-26.

116. Li E, Bestor $\mathrm{TH}$, Jaenisch R. Targeted mutation of the DNA methyltransferase gene results in embryonic lethality. Cell. 1992;69(6):915-26.

117. Okano M, Bell DW, Haber DA, Li E. DNA methyltransferases Dnmt3a and Dnmt3b are essential for de novo methylation and mammalian development. Cell. 1999;99(3):247-57.

118. Hiltunen MO, Turunen MP, Hakkinen TP, Rutanen J, Hedman M, Makinen K, Turunen AM, Aalto-Setala K, Yla-Herttuala S. DNA hypomethylation and methyltransferase expression in atherosclerotic lesions. Vasc Med. 2002;7(1): 5-11

119. Castillo-Diaz SA, Garay-Sevilla ME, Hernandez-Gonzalez MA, Solis-Martinez $\mathrm{MO}$, Zaina S. Extensive demethylation of normally hypermethylated cpg islands occurs in human atherosclerotic arteries. Int J Mol Med. 2010;26: 691-700.

120. Kim J, Kim JY, Song KS, Lee YH, Seo JS, Jelinek J, Goldschmidt-Clermont PJ, Issa JP. Epigenetic changes in estrogen receptor beta gene in atherosclerotic cardiovascular tissues and in vitro vascular senescence. Biochim Biophys Acta. 2007;1772(1):72-80

121. Rao X, Zhong J, Zhang S, Zhang Y, Yu Q, Yang P, Wang MH, Fulton DJ, Shi $\mathrm{H}$, Dong Z, Wang D, Wang CY. Loss of methyl-CpG-binding domain protein 2 enhances endothelial angiogenesis and protects mice against hind-limb ischemic injury. Circulation. 2011;123(25):2964-74.

122. Karpe PA, Tikoo K. Heat shock prevents insulin resistance induced vascular complications by augmenting angiotensin-(1-7) signalling. Diabetes. 2014; 63(3):1124-39.

123. Jamaluddin MS, Yang $X$, Wang $H$. Hyperhomocysteinemia, DNA methylation and vascular disease. Clin Chem Lab Med. 2007;45(12):1660-6.

124. Jiang YZ, Jimenez JM, Ou K, McCormick ME, Zhang LD, Davies PF. Hemodynamic disturbed flow induces differential DNA methylation of endothelial kruppel-like factor 4 (klf4) promoter in vitro and in vivo. Circ Res. 2014;115(1):32-43.

125. Dunn J, Qiu H, Kim S, Jjingo D, Hoffman R, Kim CW, Jang I, Son DJ, Kim D, Pan C, Fan Y, Jordan IK, Jo H. Flow-dependent epigenetic DNA methylation regulates gene expression and atherosclerosis. J Clin Invest. 2014;124(7): 3187-99.

126. Zhou J, Li YS, Wang KC, Chien S. Epigenetic mechanism in regulation of endothelial function by disturbed flow: induction of DNA hypermethylation by dnmt1. Cell Mol Bioeng. 2014;7(2):218-24.

127. Zhang YP, Huang YT, Huang TS, Pang W, Zhu JJ, Liu YF, Tang RZ, Zhao CR, Yao WJ, Li YS, Chien S, Zhou J. The mammalian target of rapamycin and DNA methyltransferase 1 axis mediates vascular endothelial dysfunction in response to disturbed flow. Sci Rep. 2017;7(1):14996.

\section{Publisher's Note}

Springer Nature remains neutral with regard to jurisdictional claims in published maps and institutional affiliations. 